\title{
OPTIMAL STOPPING WITH INFORMATION CONSTRAINT
}

\author{
JUKKA LEMPA
}

\begin{abstract}
We study the optimal stopping problem proposed by Dupuis and Wang in [10]. In this maximization problem of the expected present value of the exercise payoff, the underlying dynamics follow a linear diffusion. The decision maker is not allowed to stop at any time she chooses but rather on the jump times of an independent Poisson process. In [10], the authors solve this problem in the case where the underlying is a geometric Brownian motion and the payoff function is of American call option type. In the current study, we propose a mild set of conditions (covering the setup of [10]) on both the underlying and the payoff and build and use a Markovian apparatus based on the Bellman principle of optimality to solve the problem under these conditions. We also discuss the interpretation of this model as optimal timing of an irreversible investment decision under an exogenous information constraint.
\end{abstract}

\section{INTRODUCTION AND THE MAIN RESULT}

1.1. The underlying dynamics. We assume that the underlying state process $X$ is a regular linear diffusion defined on a complete filtered probability space $\left(\Omega, \mathcal{F},\left\{\mathcal{F}_{t}\right\}_{t \geq 0}, \mathbf{P}\right)$ satisfying the usual conditions and evolving on $\mathbf{R}_{+}$with the initial state $x$, see [5]. For brevity, we denote the filtration $\left\{\mathcal{F}_{t}\right\}_{t \geq 0}$ as $\mathbb{F}$. In addition, we denote as $\mathbf{P}_{x}$ the probability measure $\mathbf{P}$ conditioned on the initial state $x$ and as $\mathbf{E}_{x}$ the expectation with respect to $\mathbf{P}_{x}$. In line with most economical and financial applications, we assume that $X$ does not die inside $\mathbf{R}_{+}$, i.e., that killing of $X$ is possible only at the boundaries 0 and $\infty$. Therefore the boundaries 0 and $\infty$ are either natural, entrance, exit or regular. In the case a boundary is regular, it is assumed to be killing, see [5], pp. 18-20, for a characterization of the boundary behavior of diffusions. The life time of $X$ is defined as $\zeta:=\left\{t \geq 0: X_{t} \notin \mathbf{R}_{+}\right\}$. Now, the evolution of $X$ is completely determined by its scale function $S$ and speed measure $m$ inside $\mathbf{R}_{+}$, see [5], pp. 13-14. Furthermore, we assume that the function $S$ and the measure $m$ are both absolutely continuous with respect to the Lebesgue measure, have smooth derivatives and that $S$ is twice continuously differentiable. Under these assumptions, we know that the infinitesimal generator $\mathcal{A}: \mathcal{D}(\mathcal{A}) \rightarrow C_{b}\left(\mathbf{R}_{+}\right)$of $X$ can be expressed as $\mathcal{A}=\frac{1}{2} \sigma^{2}(x) \frac{d^{2}}{d x^{2}}+\mu(x) \frac{d}{d x}$ where the functions $\sigma$ and $\mu$ (the infinitesimal parameters of $X$ ) are related to $S$ and $m$ via the formulæ $m^{\prime}(x)=\frac{2}{\sigma^{2}(x)} e^{B(x)}$

2000 Mathematics Subject Classification. 60J60, 60G40.

Key words and phrases. optimal stopping, irreversible investment, linear diffusion, Poisson process, Bellman principle of optimality, resolvent operator.

Address. Jukka Lempa, Centre of Mathematics for Applications, University of Oslo, PO Box 1053 Blindern, NO - 0316 Oslo, e-mail: jlempa@cma.uio.no. 
and $S^{\prime}(x)=e^{-B(x)}$ for all $x \in \mathbf{R}_{+}$, where $B(x):=\int^{x} \frac{2 \mu(y)}{\sigma^{2}(y)} d y$, see [5], pp. 17. From these definitions we find that $\sigma^{2}(x)=\frac{2}{S^{\prime}(x) m^{\prime}(x)}$ and $\mu(x)=-\frac{S^{\prime \prime}(x)}{S^{\prime 2}(x) m^{\prime}(x)}$ for all $x \in \mathbf{R}_{+}$. In what follows, we assume that the functions $\mu$ and $\sigma^{2}$ are continuous. The assumption that the state space is $\mathbf{R}_{+}$is done for reasons of notational convenience. In fact, we could assume that the state space is any interval $\mathcal{I}$ in $\mathbf{R}$ and all our subsequent analysis would hold with obvious modifications. Furthermore, we denote as, respectively, $\psi_{r}$ and $\varphi_{r}$ the increasing and the decreasing solution of the ordinary second order linear differential equation $\mathcal{A} u=r u$, where $r>0$, defined on the domain of the characteristic operator of $X$ - for a characterization and fundamental properties of the minimal $r$-excessive functions $\psi_{r}$ and $\varphi_{r}$, see [5], pp. 18-20. In addition, we assume that the filtration $\mathbb{F}$ is rich enough to carry a Poisson process $N=\left(N_{t}, \mathcal{F}_{t}\right)$ with intensity $\lambda$. We call the process $N$ the signal process, and assume that $X$ and $N$ are independent.

For $r>0$, we denote as $L_{1}^{r}$ the class of real valued measurable functions $f$ on $\mathbf{R}_{+}$satisfying the condition $\mathbf{E}_{x}\left[\int_{0}^{\zeta} e^{-r s}\left|f\left(X_{s}\right)\right| d s\right]<\infty$. For a function $f \in L_{1}^{r}$, the resolvent $R_{r} f: \mathbf{R}_{+} \rightarrow \mathbf{R}$ is defined as

$$
\left(R_{r} f\right)(x)=\mathbf{E}_{x}\left[\int_{0}^{\zeta} e^{-r s} f\left(X_{s}\right) d s\right]
$$

for all $x \in \mathbf{R}_{+}$. The resolvent $R_{r}$ and the increasing and decreasing solutions $\psi_{r}$ and $\varphi_{r}$ are connected in a computationally very useful way. Indeed, we know from the literature that for a given $f \in L_{1}^{r}$ the resolvent $R_{r} f$ can be expressed as

$$
\left(R_{r} f\right)(x)=B_{r}^{-1} \varphi_{r}(x) \int_{0}^{x} \psi_{r}(y) f(y) m^{\prime}(y) d y+B_{r}^{-1} \psi_{r}(x) \int_{x}^{\infty} \varphi_{r}(y) f(y) m^{\prime}(y) d y
$$

for all $x \in \mathbf{R}_{+}$, where $B_{r}=\frac{\psi_{r}^{\prime}(x)}{S^{\prime}(x)} \varphi_{r}(x)-\frac{\varphi_{r}^{\prime}(x)}{S^{\prime}(x)} \psi_{r}(x)$ denotes the Wronskian determinant, see [5], pp. 19. We remark that the value of $B_{r}$ does not depend on the state variable $x$ but depends on the rate $r$.

1.2. The optimal stopping problems. Having the underlying dynamics set up, we formulate, following [10], our main optimal stopping problems. In comparison to the classical continuous time case, see, e.g., [2], [8], [17], and [23], see also [20], the key difference is that the decision maker is not allowed to (or cannot) exercise at any time she chooses but rather on the jump times of the independent signal process $N$. The process $N$ jumps at times $T_{1}<T_{2}<\cdots<T_{n}<\ldots$, where the intervals $\left\{T_{1}, T_{2}-T_{1}, T_{3}-T_{2}, \ldots\right\}$ are exponential IID with mean $\frac{1}{\lambda}$. We remark that by convention $T_{0}=0$ and $T_{\infty}=\infty$.

In the first optimal stopping problem, the decision maker cannot exercise at the initial time $t=0$. This means that the first jump time $T_{1}$ is the first potentially reasonable moment for her to exercise. In this 
setting, the class of admissible stopping times reads as

$$
\mathcal{T}=\left\{\tau: \text { for all } \omega \in \Omega, \tau(\omega)=T_{n}(\omega) \text { for some } n \in 1,2, \ldots, \infty\right\}
$$

Let $r>0$ be the constant discount rate and $g: \mathbf{R}_{+} \rightarrow \mathbf{R}$ the exercise payoff function which is assumed to be at least continuous. The optimal stopping problem is now to maximize the expected present value of the exercise payoff under $\left\{\mathcal{F}_{\tau}\right\}_{\tau \in \mathcal{T}}$, i.e. to determine the optimal value function

$$
V(x)=\sup _{\tau \in \mathcal{T}} \mathbf{E}_{x}\left[e^{-r \tau} g\left(X_{\tau}\right) \mathbf{1}_{\{\tau<\zeta\}}\right] .
$$

Moreover, we want to characterize the optimal stopping time $\tau^{*}$ which constitutes this value.

The second optimal stopping problem is otherwise the same as the first but now the decision maker can exercise immediately, i.e., at $t=0$. Now, the class of admissible stopping times reads as

$$
\mathcal{T}_{0}=\left\{\tau: \text { for all } \omega \in \Omega, \tau(\omega)=T_{n}(\omega) \text { for some } n \in 0,1,2, \ldots, \infty\right\}
$$

The corresponding optimal stopping problem reads as

$$
V_{0}(x)=\sup _{\tau \in \mathcal{T}_{0}} \mathbf{E}_{x}\left[e^{-r \tau} g\left(X_{\tau}\right) \mathbf{1}_{\{\tau<\zeta\}}\right],
$$

and the optimal stopping time is denoted as $\tau_{0}^{*}$. The reason for the simultaneous introduction of these problems is mostly technical, as their analyzes will be intertwined.

1.3. Main result and discussion. In the literature of optimal stopping problems of the form (1.4), the incorporated exogenous Poisson processes, or more general renewal processes, appear in various roles. In principle, this process can affect three different components of the problem, namely the parameters of the underlying dynamics, the payoff structure, and/or the set of admissible exercise times. An example of models where the underlying is affected fall into the class of regime switching models, where the changes in the drift and volatility are triggered exogenously, see, e.g., [12], [14], and [16]. The payoff structure is affected, for example, in a real option approach to the technology adaption of a value maximizing firm, where new technologies emerge according to the jumps of the exogenous innovation process, see, e.g., [3], [4], and [6]. More precisely, the exogenous innovation process affects the firms exit (or entrance) strategy as the adoption of new technologies changes the expected present value of the cash flow accrued from the production.

The setup of the study at hand serves as an example of a class of problems where the set of admissible stopping times is affected by the exogenous signal process. This class of problems was first proposed in [10], where the authors solve the special case of perpetual American call with underlying geometric Brownian 
motion. The same signal process setting was adopted in [13], where the authors generalize the results of [24] for stopping geometric Brownian motion at its maximum. Generally speaking, the process $N$ can be seen as an exogenous constraint on the decision makers ability to exercise. This constraint has different interpretations. In [10], the authors propose, along the lines of [22], that the signal process $N$ reflects liquidity effects, i.e., the process $N$ dictates the times at which the asset is available to trade. Following [13], we remark that the considered optimal stopping problem can also be seen as a valuation problem of a randomized version of a perpetual Bermudan option, where contract allows the holder to exercise only at the jump times of the process $N$. The process $N$ can also be seen as an information constraint. Now, the holder is able exercise at all times but can observe the return process only at the jump times of $N$. The holder is forced to make her timing decision based on partial information on $X$ where the signal process $N$ stipulates the exogenous restriction on the information available to her. In this setting, the sample paths observed by the decision maker are pure jump trajectories with jumps at Poissonian times $T_{i}$ and remaining constant in between, see Figure 1.

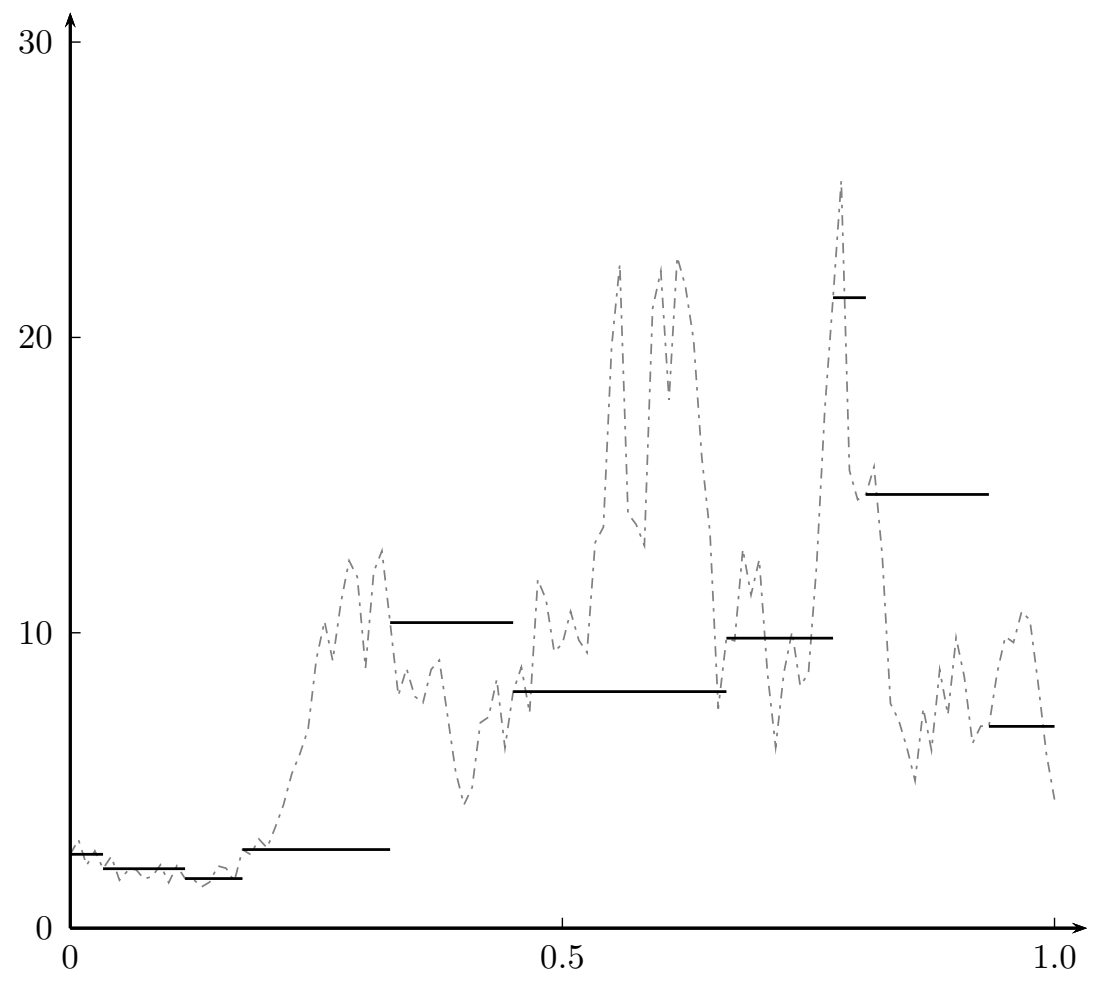

Figure 1. Picture of a possible realization of the underlying diffusion $X$ (grey trajectory) and the pure jump path determined by the exponentially arriving observations of $X$ (black trajectory). In this realization, the high return around $t=0.6$ is not observed and therefore missed by the investor 
Our objective is to prove a generalization of the main result in [10]. This generalization, which is new to our best knowledge, is formulated in the next theorems.

Theorem 1.1. Assume that the upper boundary $\infty$ is natural and the lower boundary 0 is natural, entrance, exit or killing for the underlying $X$. Assume that the payoff $g$ is continuous and in $L_{1}^{r}$. Furthermore, assume that there is a unique state $\hat{x}$ which maximizes the function $x \mapsto \frac{g(x)}{\psi_{r}(x)}$, that this function is nondecreasing on $(0, \hat{x})$ and nonincreasing on $(\hat{x}, \infty)$, and that it satisfies the limiting conditions $\lim _{x \rightarrow 0+} \frac{g(x)}{\psi_{r}(x)}=\lim _{x \rightarrow \infty} \frac{g(x)}{\psi_{r}(x)}=0$. Then the threshold $x^{*}<\hat{x}$ characterized uniquely by the condition

$$
\psi_{r}\left(x^{*}\right) \int_{x^{*}}^{\infty} \varphi_{r+\lambda}(y) g(y) m^{\prime}(y) d y=g\left(x^{*}\right) \int_{x^{*}}^{\infty} \varphi_{r+\lambda}(y) \psi_{r}(y) m^{\prime}(y) d y
$$

gives rise to the optimal stopping region $\left[x^{*}, \infty\right)$ for the optimal stopping problems (1.4) and (1.6). Moreover, the optimal value functions $V \in C^{2}\left(\mathbf{R}_{+}\right)$and $V_{0} \in C\left(\mathbf{R}_{+}\right)$can be written as

$$
V(x)=\lambda\left(R_{r+\lambda} V_{0}\right)(x)= \begin{cases}\lambda\left(R_{r+\lambda} g\right)(x)+\frac{g\left(x^{*}\right)-\lambda\left(R_{r+\lambda} g\right)\left(x^{*}\right)}{\varphi_{r+\lambda}\left(x^{*}\right)} \varphi_{r+\lambda}(x), & x \geq x^{*}, \\ \frac{g\left(x^{*}\right)}{\psi_{r}\left(x^{*}\right)} \psi_{r}(x), & x<x^{*}\end{cases}
$$

and

$$
V_{0}(x)= \begin{cases}g(x), & x \geq x^{*}, \\ \frac{g\left(x^{*}\right)}{\psi_{r}\left(x^{*}\right)} \psi_{r}(x), & x<x^{*}\end{cases}
$$

Theorem 1.2. Assume that the lower boundary 0 is natural and the upper boundary $\infty$ is natural, entrance, exit or killing for the underlying $X$. Assume that the payoff $g$ is continuous and in $L_{1}^{r}$. Furthermore, assume that there is a unique state $\tilde{x}$ which maximizes the function $x \mapsto \frac{g(x)}{\varphi_{r}(x)}$, that this function is nondecreasing on $(0, \tilde{x})$ and nonincreasing on $(\tilde{x}, \infty)$, and that it satisfies the limiting conditions $\lim _{x \rightarrow 0+} \frac{g(x)}{\varphi_{r}(x)}=\lim _{x \rightarrow \infty} \frac{g(x)}{\varphi_{r}(x)}=0$. Then the threshold $x^{\dagger}>\tilde{x}$ characterized uniquely by the condition

$$
\varphi_{r}\left(x^{\dagger}\right) \int_{0}^{x^{\dagger}} \psi_{r+\lambda}(y) g(y) m^{\prime}(y) d y=g\left(x^{\dagger}\right) \int_{0}^{x^{\dagger}} \psi_{r+\lambda}(y) \varphi_{r}(y) m^{\prime}(y) d y
$$

gives rise to the optimal stopping region $\left(0, x^{*}\right]$ for the optimal stopping problems (1.4) and (1.6). Moreover, the optimal value functions $V \in C^{2}\left(\mathbf{R}_{+}\right)$and $V_{0} \in C\left(\mathbf{R}_{+}\right)$can be written as

$$
V(x)=\lambda\left(R_{r+\lambda} V_{0}\right)(x)= \begin{cases}\frac{g\left(x^{\dagger}\right)}{\varphi_{r}\left(x^{\dagger}\right)} \varphi_{r}(x), & x>x^{\dagger}, \\ \lambda\left(R_{r+\lambda} g\right)(x)+\frac{g\left(x^{\dagger}\right)-\lambda\left(R_{r+\lambda} g\right)\left(x^{\dagger}\right)}{\psi_{r+\lambda}\left(x^{\dagger}\right)} \psi_{r+\lambda}(x), & x \leq x^{\dagger},\end{cases}
$$


and

$$
V_{0}(x)= \begin{cases}\frac{g\left(x^{\dagger}\right)}{\varphi_{r}\left(x^{\dagger}\right)} \varphi_{r}(x), & x>x^{\dagger} \\ g(x), & x \leq x^{\dagger}\end{cases}
$$

We make a few remarks on the assumptions of Theorems 1.1 and 1.2. It is interesting to note that the existence of a unique optimal stopping threshold can be returned essentially to the monotonicity properties of the function $x \mapsto \frac{g(x)}{\psi_{r}(x)}$ (or $\left.x \mapsto \frac{g(x)}{\varphi_{r}(x)}\right)$. In comparison to [2], Theorem 3, we make additional assumptions on the limiting behavior of these functions and on the integrability of the payoff $g$. However, these additional assumptions are not very restrictive from the applications point of view. In this sense, it is interesting to note that the restriction of the admissible stopping times from the entire set of $\mathbb{F}$-stopping times to random times with exponential arrivals does not result into any severe additional restrictions on the underlying $X$ and the payoff $g$. As was mentioned earlier, the function $\psi_{r}$ is an increasing solution of the ordinary second order differential equation $(\mathcal{A}-r) \psi_{r}=0$ satisfying suitable boundary conditions. Even though it is not possible solve this ODE explicitly except in special cases, there are well developed methods for solving such equations numerically. This makes the numerical verification of the monotonicity and limiting conditions of the function $x \mapsto \frac{g(x)}{\psi_{r}(x)}$ plausible; the same applies naturally for Theorem 1.2 and the function $x \mapsto \frac{g(x)}{\varphi_{r}(x)}$.

The reminder of the paper is organized as follows. In Section 2, we carry out a proof for Theorem 1.1 by first deriving the candidates for the solutions and then verifying that these candidates are the actual solutions. We remark that the proof of Theorem 1.2 has a completely analogous proof to the one of Theorem 1.1 and will therefore be omitted. In Section 2, we also study the asymptotics of the solutions with respect to the parameter $\lambda$. In Section 3, we illustrate our results with four explicit examples including the case of [10]. Section 4 concludes the study.

\section{Proof of the main Result}

2.1. Some preliminary analysis. We start the preliminary analysis by proving some useful properties of harmonic functions.

Lemma 2.1. Let $f \in C\left(\mathbf{R}_{+}\right)$and $r>0$. If, in addition, there exists $\lambda>0$ and an open $A \subseteq \mathbf{R}_{+}$such that $\lambda\left(R_{r+\lambda} f\right)(x)=f(x)$ for all $x \in A$, then $(\mathcal{A}-r) f(x)=0$ for all $x \in A$. On the contrary, if

(a) $f$ is r-harmonic and the boundaries 0 and $\infty$ are natural, then $\lambda\left(R_{r+\lambda} f\right)(x)=f(x)$,

(b) $\infty$ is natural and 0 is entrance, exit or killing, then $\lambda\left(R_{r+\lambda} \psi_{r}\right)(x)=\psi_{r}(x)$ and $\lambda\left(R_{r+\lambda} \varphi_{r}\right)(x)=$ $\varphi_{r}(x)-A_{1} \varphi_{r+\lambda}(x)$, where $A_{1}=\lim _{z \rightarrow 0} \frac{\varphi_{r}(z)}{\varphi_{r+\lambda}(z)}>0$, 
(c) 0 is natural and $\infty$ is entrance, exit or killing, then $\lambda\left(R_{r+\lambda} \varphi_{r}\right)(x)=\varphi_{r}(x)$ and $\lambda\left(R_{r+\lambda} \psi_{r}\right)(x)=$ $\psi_{r}(x)-A_{2} \psi_{r+\lambda}(x)$, where $A_{2}=\lim _{z \rightarrow \infty} \frac{\psi_{r}(z)}{\psi_{r+\lambda}(z)}>0$,

(d) $f$ is $r$-harmonic and the boundaries 0 and $\infty$ are entrance, exit or killing, then $\lambda\left(R_{r+\lambda} f\right)(x)<f(x)$, for all $\lambda>0$ and $x \in \mathbf{R}_{+}$.

Proof. Assume that there exists $\lambda>0$ and an open $A \subseteq \mathbf{R}_{+}$such that $\lambda\left(R_{r+\lambda} f\right)(x)=f(x)$ for all $x \in A$. Now, using the representation (1.2) and the harmonicity properties of $\psi_{r+\lambda}$ and $\varphi_{r+\lambda}$, it is a matter of differentiation to show that

$$
\begin{aligned}
(\mathcal{A}-r)\left(R_{r+\lambda} f\right)(x) & =B_{r+\lambda}^{-1}(\mathcal{A}-r) \varphi_{r+\lambda}(x) \int_{0}^{x} \psi_{r+\lambda}(y) f(y) m^{\prime}(y) d y \\
& +B_{r+\lambda}^{-1}(\mathcal{A}-r) \psi_{r+\lambda}(x) \int_{x}^{\infty} \varphi_{r+\lambda}(y) f(y) m^{\prime}(y) d y-f(x) \\
& =B_{r+\lambda}^{-1} \lambda \varphi_{r+\lambda}(x) \int_{0}^{x} \psi_{r+\lambda}(y) f(y) m^{\prime}(y) d y \\
& +B_{r+\lambda}^{-1} \lambda \psi_{r+\lambda}(x) \int_{x}^{\infty} \varphi_{r+\lambda}(y) f(y) m^{\prime}(y) d y-f(x) \\
& =\lambda\left(R_{r+\lambda} f\right)(x)-f(x)=0,
\end{aligned}
$$

for all $x \in A$. Since $f(x)=\lambda\left(R_{r+\lambda} f\right)(x)$ on $A$ and $A$ is open, the claim follows.

To prove the remaining claims, let $f$ be $r$-harmonic. Then, in particular, $f$ is twice continuously differentiable because we have assumed that $\mu$ and $\sigma$ are continuous. Consider the Markov times $S_{n}: n \mapsto \inf \{t \geq$ $\left.0: X_{t} \notin\left(n^{-1}, n\right)\right\}$ for $n \geq 1$. Now, Dynkin's formula, see [11], pp. 131-133, yields

$$
\begin{aligned}
\mathbf{E}_{x}\left[e^{-(r+\lambda)\left(S_{n} \wedge k\right)} f\left(X_{S_{n} \wedge k}\right)\right] & =f(x)+\underbrace{\mathbf{E}_{x}\left[\int_{0}^{S_{n} \wedge k} e^{-(r+\lambda) s}(\mathcal{A}-r) f\left(X_{s}\right) d s\right]}_{=0} \\
& -\lambda \mathbf{E}_{x}\left[\int_{0}^{S_{n} \wedge k} e^{-(r+\lambda) s} f\left(X_{s}\right) d s\right]
\end{aligned}
$$

for all $k \in \mathbf{N}$. Since $e^{-(r+\lambda)\left(S_{n} \wedge k\right)} f\left(X_{S_{n} \wedge k}\right) \leq \sup _{z \in\left[n^{-1}, n\right]} f(z)<\infty$ for a fixed $n$ and $f$ is non-negative, we can use bounded (monotone) convergence pass to the limit $k \rightarrow \infty$ on the left (right) hand side of (2.1) and obtain

$$
\mathbf{E}_{x}\left[e^{-(r+\lambda) S_{n}} f\left(X_{S_{n}}\right)\right]=f(x)-\lambda \mathbf{E}_{x}\left[\int_{0}^{S_{n}} e^{-(r+\lambda) s} f\left(X_{s}\right) d s\right] .
$$

Since $S_{n}$ is non-decreasing and $S_{n} \rightarrow \zeta$ as $n \rightarrow \infty$, monotone convergence yields

$$
\lambda \mathbf{E}_{x}\left[\int_{0}^{S_{n}} e^{-(r+\lambda) s} f\left(X_{s}\right) d s\right] \rightarrow \lambda\left(R_{r+\lambda} f\right)(x), n \rightarrow \infty .
$$


Let $x \in\left(n^{-1}, n\right)$ for a given $n \geq 2$. We know, see, e.g., [18], that

$$
\begin{aligned}
\mathbf{E}_{x}\left[e^{-(r+\lambda) S_{n}} f\left(X_{S_{n}}\right)\right] & =\mathbf{E}_{x}\left[e^{-(r+\lambda) \tau_{n}} \mathbf{1}_{\left\{\tau_{n}<\tau_{n}-1\right\}}\right] f(n)+\mathbf{E}_{x}\left[e^{-(r+\lambda) \tau_{n}-1} \mathbf{1}_{\left\{\tau_{n}>\tau_{n}-1\right.}\right] f\left(n^{-1}\right) \\
& =\frac{\varphi_{r+\lambda}(n) f\left(n^{-1}\right)-\varphi_{r+\lambda}\left(n^{-1}\right) f(n)}{\varphi_{r+\lambda}(n) \psi_{r+\lambda}\left(n^{-1}\right)-\varphi_{r+\lambda}\left(n^{-1}\right) \psi_{r+\lambda}(n)} \psi_{r+\lambda}(x) \\
& +\frac{\psi_{r+\lambda}\left(n^{-1}\right) f(n)-\psi_{r+\lambda}(n) f\left(n^{-1}\right)}{\varphi_{r+\lambda}(n) \psi_{r+\lambda}\left(n^{-1}\right)-\varphi_{r+\lambda}\left(n^{-1}\right) \psi_{r+\lambda}(n)} \varphi_{r+\lambda}(x),
\end{aligned}
$$

where the first hitting time $\tau_{y}=\inf \left\{t \geq 0: X_{t}=y\right\}$. To proceed, we prove the claim (b)-claims (a), (c), and $(d)$ are treated in the same manner. Consider first the case $f=\psi_{r}$. We rewrite (2.2) as

$$
\begin{aligned}
\mathbf{E}_{x}\left[e^{-(r+\lambda) S_{n}} \psi_{r}\left(X_{S_{n}}\right)\right] & =\frac{\psi_{r}(n)}{\psi_{r+\lambda}(n)} \underbrace{\frac{1-\frac{\psi_{r}\left(n^{-1}\right)}{\psi_{r}(n)} \frac{\varphi_{r+\lambda}(n)}{\varphi_{r+\lambda}\left(n^{-1}\right)}}{1-\frac{\psi_{r+\lambda}\left(n^{-1}\right)}{\psi_{r+\lambda}(n)} \frac{\varphi_{r+\lambda}(n)}{\varphi_{r+\lambda}\left(n^{-1}\right)}}}_{:=a_{1}(n)} \psi_{r+\lambda}(x) \\
& +\frac{\psi_{r}\left(n^{-1}\right)}{\varphi_{r+\lambda}\left(n^{-1}\right)} \underbrace{\frac{1-\frac{\psi_{r+\lambda}\left(n^{-1}\right)}{\psi_{r+\lambda}(n)} \frac{\psi_{r}(n)}{\psi_{r}\left(n{ }^{-1}\right)}}{1-\frac{\psi_{r+\lambda}\left(n^{-1}\right)}{\psi_{r+\lambda}(n)} \frac{\varphi_{r+\lambda}(n)}{\varphi_{r+\lambda}\left(n^{-1}\right)}}}_{:=a_{2}(n)} \varphi_{r+\lambda}(x) .
\end{aligned}
$$

Since $\psi \cdot(n) \rightarrow \infty$ as $n \rightarrow \infty$, the monotonicity properties of $\psi$. and $\varphi$. imply that $a_{1}(n) \rightarrow 1$ as $n \rightarrow \infty$ - see [5], pp. 18-20, for the limiting behavior of $\psi$. and $\varphi$.. On the other hand, since

$$
\frac{\psi_{r}\left(n^{-1}\right)}{\psi_{r}(n)}=\mathbf{E}_{n^{-1}}\left[e^{-r \tau_{n}}\right] \geq \mathbf{E}_{n^{-1}}\left[e^{-(r+\lambda) \tau_{n}}\right]=\frac{\psi_{r+\lambda}\left(n^{-1}\right)}{\psi_{r+\lambda}(n)}
$$

we find using the assumed boundary behavior that $\lim _{\sup _{n \rightarrow \infty}} a_{2}(n) \leq 1$. Moreover, we observe from this inequality that the function $x \mapsto \frac{\psi_{r}(x)}{\psi_{r+\lambda}(x)}$ is decreasing. Now, since $\infty$ is natural (implying that $\lim _{n \rightarrow \infty} \frac{\psi_{.}{ }^{\prime}(n)}{S^{\prime}(n)}=$ $\infty)$, we find by first using l'Hôpital's rule twice and then the identities $(\mathcal{A}-r) \psi_{r}=(\mathcal{A}-(r+\lambda)) \psi_{r+\lambda}=0$ coupled with the definition of $S^{\prime}$ that

$$
\lim _{n \rightarrow \infty} \frac{\psi_{r}(n)}{\psi_{r+\lambda}(n)}=\lim _{n \rightarrow \infty} \frac{\frac{\psi_{r}{ }^{\prime}(n)}{S^{\prime}(n)}}{\frac{\psi_{r+\lambda^{\prime}(n)}}{S^{\prime}(n)}}=\lim _{n \rightarrow \infty} \frac{S^{\prime}(n) \psi_{r}^{\prime \prime}(n)-S^{\prime \prime}(n) \psi_{r}{ }^{\prime}(n)}{S^{\prime}(n) \psi_{r+\lambda}{ }^{\prime \prime}(n)-S^{\prime \prime}(n) \psi_{r+\lambda}(n)}=\frac{r}{r+\lambda} \lim _{n \rightarrow \infty} \frac{\psi_{r}(n)}{\psi_{r+\lambda}(n)} .
$$

This implies that the limiting value must be zero. Finally, the assumed boundary behavior implies that also $\frac{\psi_{r}\left(n^{-1}\right)}{\varphi_{r+\lambda}\left(n^{-1}\right)}$ and, consequently, $\mathbf{E}_{x}\left[e^{-(r+\lambda) S_{n}} \psi_{r}\left(X_{S_{n}}\right)\right] \rightarrow 0$ as $n \rightarrow \infty$. This proves the claim on $\psi_{r}$. 
Consider now the case $f=\varphi_{r}$. We rewrite $(2.2)$ as

$$
\begin{aligned}
\mathbf{E}_{x}\left[e^{-(r+\lambda) S_{n}} \varphi_{r}\left(X_{S_{n}}\right)\right] & =\frac{\varphi_{r}(n)}{\psi_{r+\lambda}(n)} \underbrace{\frac{1-\frac{\varphi_{r+\lambda}(n)}{\varphi_{r+\lambda}\left(n^{-1}\right)} \frac{\varphi_{r}\left(n^{-1}\right)}{\varphi_{r}(n)}}{1-\frac{\psi_{r+\lambda}\left(n^{-1}\right)}{\psi_{r+\lambda}(n)} \frac{\varphi_{r+\lambda}(n)}{\varphi_{r+\lambda}\left(n^{-1}\right)}}}_{:=b_{1}(n)} \psi_{r+\lambda}(x) \\
& +\frac{\varphi_{r}\left(n^{-1}\right)}{\varphi_{r+\lambda}\left(n^{-1}\right)} \underbrace{\frac{1-\frac{\psi_{r+\lambda}\left(n^{-1}\right)}{\psi_{r+\lambda}(n)} \frac{\varphi_{r}(n)}{\varphi_{r}\left(n^{-1}\right)}}{1-\frac{\psi_{r+\lambda}\left(n^{-1}\right)}{\psi_{r+\lambda}(n)} \frac{\varphi_{r+\lambda}(n)}{\varphi_{r+\lambda}\left(n^{-1}\right)}}}_{:=b_{2}(n)} \varphi_{r+\lambda}(x) .
\end{aligned}
$$

Similarly to the previous case, we find that $\limsup _{n \rightarrow \infty} b_{1}(n) \leq 1$ and $\lim _{n \rightarrow \infty} b_{2}(n)=1$. Since $\frac{\varphi_{r}(n)}{\psi_{r+\lambda}(n)} \rightarrow 0$ as $n \rightarrow \infty$, we conclude, analogously to (2.4), that

$$
\lim _{n \rightarrow \infty} \mathbf{E}_{x}\left[e^{-(r+\lambda) S_{n}} \varphi_{r}\left(X_{S_{n}}\right)\right]=\lim _{z \rightarrow 0} \frac{\varphi_{r}(z)}{\varphi_{r+\lambda}(z)} \varphi_{r+\lambda}(x)>0,
$$

proving the claim on $\varphi_{r}$.

To illustrate the conclusion of Lemma 2.1, consider first a regular diffusion process $X$ with the differential generator $\mathcal{A}=\frac{1}{2} x^{4} \frac{d^{2}}{d x^{2}}$ and the initial state $x>0$. This process can be identified as the reciprocal of a Bessel $(3)$ process (aka a CEV process, see, e.g., [15]). The origin is natural and $\infty$ is an entrance boundary for $X$. Now, the functions $\psi_{r}$ and $\varphi_{r}$ read as $\psi_{r}(x)=x \exp \left(-\sqrt{2 r} x^{-1}\right)$ and $\varphi_{r}(x)=x \sinh \left(\sqrt{2 r} x^{-1}\right)$. Moreover, the Wronskian $B_{r}=\sqrt{2 r}$. Using (1.2), it is a matter of integration to show that

$$
\lambda\left(R_{r+\lambda} \psi_{r}\right)(x)=\psi_{r}(x)\left[1-\exp \left(-\frac{\sqrt{2(r+\lambda)}-\sqrt{2 r}}{x}\right)\right]=\psi_{r}(x)-\psi_{r+\lambda}(x) \text { and } \lambda\left(R_{r+\lambda} \varphi_{r}\right)(x)=\varphi_{r}(x)
$$

In particular, we note that $\lambda\left(R_{r+\lambda} \psi_{r}\right)(x)=0$ as $\lambda \rightarrow 0$. As another example, let $X$ be a standard Brownian motion killed in the origin with the initial state $x>0$. Now, the boundary $\infty$ is natural. In this case, the functions $\psi_{r}$ and $\varphi_{r}$ read as $\psi_{r}(x)=\sinh (\sqrt{2 r} x)$ and $\varphi_{r}(x)=\exp (-\sqrt{2 r} x)$. The Wronskian $B_{r}=\sqrt{2 r}$. Now, using (1.2) it is straightforward to compute that

$$
\lambda\left(R_{r+\lambda} \psi_{r}\right)(x)=\psi_{r}(x) \quad \text { and } \quad \lambda\left(R_{r+\lambda} \varphi_{r}\right)(x)=\varphi_{r}(x)-\varphi_{r+\lambda}(x)
$$

This time we find that $\lambda\left(R_{r+\lambda} \varphi_{r}\right)(x)=0$ as $\lambda \rightarrow 0$.

The assumptions of Theorem 1.1 restraining the choice of the payoff function $g$ and the underlying $X$ are relatively weak and easy to verify, at least numerically. We know from [2] that the ratio function $x \mapsto \frac{g(x)}{\psi_{r}(x)}$ and its monotonicity properties play a key role in the classical continuous time case. In the current setting, it not the ratio $x \mapsto \frac{g(x)}{\psi_{r}(x)}$ but something at least formally quite similar that characterizes the optimal stopping 
rule. To make a precise statement, define the functions $I: \mathbf{R}_{+} \rightarrow \mathbf{R}$ and $J: \mathbf{R}_{+} \rightarrow \mathbf{R}$ as

$$
\begin{aligned}
& I(x)=\int_{x}^{\infty} \varphi_{r+\lambda}(y) g(y) m^{\prime}(y) d y, \\
& J(x)=\int_{x}^{\infty} \varphi_{r+\lambda}(y) \psi_{r}(y) m^{\prime}(y) d y,
\end{aligned}
$$

for all $x \in \mathbf{R}_{+}$. We remark that it follows from the proof of Lemma 2.1 that the function $J$ is well-defined. The ratio function $x \mapsto \frac{I(x)}{J(x)}$ will play a key role when proving Theorem 1.1. The next lemma provides us with the required monotonicity properties of this function.

Lemma 2.2. Let the assumptions of Theorem 1.1 hold. Then there is a unique state $x^{*}<\hat{x}$ that maximizes the function $x \mapsto \frac{I(x)}{J(x)}$. Moreover, the function $x \mapsto \frac{I(x)}{J(x)}$ is nondecreasing on $\left(0, x^{*}\right)$ and nonincreasing on $\left(0, x^{*}\right)$.

Proof. First, straightforward differentiation yields the condition

$$
\frac{d}{d x}\left(\frac{I(x)}{J(x)}\right) \gtreqless 0 \text { if and only if } \psi_{r}(x) I(x) \gtreqless g(x) J(x) \text {. }
$$

Let $x \geq \hat{x}$. Since the function $x \mapsto \frac{g(x)}{\psi_{r}(x)}$ is nonincreasing on $(\hat{x}, \infty)$, we find that

$$
\begin{aligned}
\psi_{r}(x) I(x)-g(x) J(x) & =\psi_{r}(x) \int_{x}^{\infty} \varphi_{r+\lambda}(y) \frac{g(y)}{\psi_{r}(y)} \psi_{r}(y) m^{\prime}(y) d y-g(x) J(x) \\
& <\left(\psi_{r}(x) \frac{g(x)}{\psi_{r}(x)}-g(x)\right) J(x)=0 .
\end{aligned}
$$

Furthermore, since the function $x \mapsto \frac{g(x)}{\psi_{r}(x)}$ tends to 0 as $x \rightarrow \infty$, we conclude using the condition (2.8) that the function $x \mapsto \frac{I(x)}{J(x)}$ is nonincreasing on $(\hat{x}, \infty)$ and tends to 0 as $x \rightarrow \infty$. On the other hand, since $\lim _{x \rightarrow 0+} \frac{g(x)}{\psi_{r}(x)}=0$ and $\frac{I(\hat{x})}{J(\hat{x})}>0$, we find using the condition (2.8) that the function $x \mapsto \frac{I(x)}{J(x)}$ must have at least one interior maximum $x^{*}<\hat{x}$. Finally, since $\frac{g\left(x^{*}\right)}{\psi_{r}\left(x^{*}\right)}=\frac{I\left(x^{*}\right)}{J\left(x^{*}\right)}, x \mapsto \frac{I(x)}{J(x)}$ is continuously differentiable, and $x \mapsto \frac{g(x)}{\psi_{r}(x)}$ nondecreasing on $(0, \hat{x})$, we conclude, again using (2.8), that the maximum $x^{*}$ is unique.

In Lemma 2.2 we proved that the function $x \mapsto \frac{I(x)}{J(x)}$ has a unique global maximum $x^{*}$. We remark that $x^{*}$ is the unique state satisfying the condition

$$
\psi_{r}\left(x^{*}\right) \int_{x^{*}}^{\infty} \varphi_{r+\lambda}(y) g(y) m^{\prime}(y) d y=g\left(x^{*}\right) \int_{x^{*}}^{\infty} \varphi_{r+\lambda}(y) \psi_{r}(y) m^{\prime}(y) d y
$$

2.2. Necessary conditions. We start the analysis of the optimal stopping problems (1.4) and (1.6) by deriving necessary conditions for the existence of a unique optimal solution. As a result, we find unique candidates 
for the optimal values $V$ and $V_{0}$ and the associated optimal stopping rules. We derive the candidates using two different approaches.

2.2.1. Via the resolvent semigroup. In this subsection we derive the candidates for optimal characteristics with a direct application of Bellman principle of optimality. We use the variational inequality formulation of Bellman principle, see, e.g., [19]. Furthermore, we exploit the close connection of the resolvent semigroup and exponentially distributed random times. Denote as $G$ and $G_{0}$ the candidates for the optimal values of the problems (1.4) and (1.6), respectively. Given the time homogeneity of the underlying $X$ and the constant jump rate of the signal process $N$, we make the ansatz that the optimal continuation region is an interval $\left(0, y^{*}\right)$ in both problems. The associated candidates for the optimal stopping times are the first exit times $T_{N_{y^{*}}}$, where $N_{y^{*}}=\inf \left\{n \geq 1: X_{T_{n}} \geq y^{*}\right\}$, in (1.4) and $T_{N_{y^{*}}^{0}}$, where $N_{y^{*}}^{0}=\inf \left\{n \geq 0: X_{T_{n}} \geq y^{*}\right\}$, in (1.6). In the problem (1.6), the decision maker chooses between two actions at every jump time $T_{i}$, $i=0,1, \ldots$ : she either exercises or waits. If she chooses to exercise, she gets the payoff $g(x)$. On the other hand, if she waits, the expected discounted value accrued from this choice is determined by the expectation $\mathbf{E}_{x}\left[e^{-r U} G_{0}\left(X_{U}\right)\right]=\lambda\left(R_{r+\lambda} G_{0}\right)(x)$, where $U$ is an independent, exponentially distributed random time with mean $\frac{1}{\lambda}$. Given these arguments, we assume that the candidate $G_{0}$ satisfies the variational inequality

$$
G_{0}(x)=\max \left\{g(x), \lambda\left(R_{r+\lambda} G_{0}\right)(x)\right\}
$$

for all $x \in \mathbf{R}_{+}$, see also [10], Remark 3, p. 144. To analyze (2.10), we remark that by assumption the candidate $G_{0}$ coincides with the payoff $g$ on the exercise region $\left[y^{*}, \infty\right)$ and satisfies the condition $G_{0}(x)=\lambda\left(R_{r+\lambda} G_{0}\right)(x)$ on the continuation region $\left(0, y^{*}\right)$. Using Lemma 2.1 we find that $G_{0}(x)=c_{1} \psi_{r}(x)+c_{2} \varphi_{r}(x)$ for all $x \in\left(0, y^{*}\right)$. Since we are looking for a solution that is bounded in the origin, we find that $c_{2}=0$. Moreover, since the value function is continuous, we conclude that $G_{0}(x)=\frac{g\left(y^{*}\right)}{\psi_{r}\left(y^{*}\right)} \psi_{r}(x)$ for all $x \in\left(0, y^{*}\right)$.

Next we characterize the optimal exercise threshold $y^{*}$ such that the variational inequality $(2.10)$ is satisfied. To this end, we find using Lemma 2.1 and the representation (1.2) that

$$
\begin{aligned}
G_{0}(x) & =\frac{g\left(y^{*}\right)}{\psi_{r}\left(y^{*}\right)} \psi_{r}(x) \\
& =\lambda\left(R_{r+\lambda} \frac{g\left(y^{*}\right)}{\psi_{r}\left(y^{*}\right)} \psi_{r}\right)(x) \\
& =\lambda\left(R_{r+\lambda} G_{0}\right)(x)+\frac{\lambda}{B_{r+\lambda}}\left(\psi_{r+\lambda}(x) \int_{y^{*}}^{\infty} \varphi_{r+\lambda}(z)\left(\frac{g\left(y^{*}\right)}{\psi_{r}\left(y^{*}\right)} \psi_{r}(z)-g(z)\right) m^{\prime}(z) d z\right),
\end{aligned}
$$

for all $x<y^{*}$. By comparing the expression (2.11) to Lemma 2.2 and the expression (2.9), we readily find that in (2.11) the last integral term vanishes and, consequently, the balance condition in (2.10) is satisfied if 
and only if $y^{*}=x^{*}$, where $x^{*}$ is defined in (2.9). Now, the candidate $G_{0}$ can be expressed as

$$
G_{0}(x)= \begin{cases}g(x), & x \geq x^{*}, \\ \frac{g\left(x^{*}\right)}{\psi_{r}\left(x^{*}\right)} \psi_{r}(x), & x<x^{*} .\end{cases}
$$

We turn to the determination of the candidate $G$. In the problem (1.4) immediate exercise is not allowed, so the decision maker must first wait an exponentially distributed period with mean $\frac{1}{\lambda}$ to make any action. After that she will face the same choice as in the problem (1.6), i.e., the choice of either exercising or postponing the exercise for another exponentially distributed random period. This argument gives rise to the balance condition

$$
G(x)=\lambda\left(R_{r+\lambda} G_{0}\right)(x),
$$

for all $x \in \mathbf{R}_{+}$, see also [10], Remark 3, p. 144. Assume that $x^{*}$ gives rise to the optimal exercise rule also in the problem (1.4). Then we find using the conditions (2.11) and (2.13) that

$$
G(x)= \begin{cases}\lambda\left(R_{r+\lambda} G_{0}\right)(x), & x \geq x^{*} \\ \frac{g\left(x^{*}\right)}{\psi_{r}\left(x^{*}\right)} \psi_{r}(x), & x<x^{*}\end{cases}
$$

Let $x \geq x^{*}$. Then using Lemma 2.1 and representation (1.2), we find that

$$
\begin{aligned}
G(x) & =\lambda\left(R_{r+\lambda} g\right)(x)+\frac{\lambda}{B_{r+\lambda}}\left(\int_{0}^{x^{*}} \psi_{r+\lambda}(z)\left(\frac{g\left(x^{*}\right)}{\psi_{r}\left(x^{*}\right)} \psi_{r}(z)-g(z)\right) m^{\prime}(z) d z\right) \frac{\varphi_{r+\lambda}(x)}{\varphi_{r+\lambda}\left(x^{*}\right)} \\
& =\lambda\left(R_{r+\lambda} g\right)(x)+\frac{g\left(x^{*}\right)-\lambda\left(R_{r+\lambda} g\right)\left(x^{*}\right)}{\varphi_{r+\lambda}\left(x^{*}\right)} \varphi_{r+\lambda}(x),
\end{aligned}
$$

and, consequently, that the candidate $G$ can be written as

$$
G(x)= \begin{cases}\lambda\left(R_{r+\lambda} g\right)(x)+\frac{g\left(x^{*}\right)-\lambda\left(R_{r+\lambda} g\right)\left(x^{*}\right)}{\varphi_{r+\lambda}\left(x^{*}\right)} \varphi_{r+\lambda}(x), & x \geq x^{*} \\ \frac{g\left(x^{*}\right)}{\psi_{r}\left(x^{*}\right)} \psi_{r}(x), & x<x^{*}\end{cases}
$$

We have now derived unique candidates $\left(G, x^{*}\right)$ given by $(2.14)$ and $(2.9)$, and $\left(G_{0}, x^{*}\right)$ given by $(2.12)$ and (2.9) for the optimal characteristics of the problems (1.4) and (1.6), respectively, under the assumptions of Theorem 1.1. Since $x^{*}<\hat{x}=\operatorname{argmax}\left\{\frac{g}{\psi_{r}}\right\}$, we conclude that the candidate $G_{0}$ is only continuous over the boundary $x^{*}$, cf. [2]. On the other hand, since the functions $\mu$ and $\sigma$ are assumed to be continuous and $G_{0}$ is continuous, we conclude using Lemma 2.1 that the candidate $G$ is twice continuously differentiable. 
2.2.2. Via a free boundary problem. In the previous subsection we derived the candidates $\left(G, x^{*}\right)$ and $\left(G_{0}, x^{*}\right)$ for the optimal characteristics of the problems (1.4) and (1.6) using the resolvent operator. These candidates can also be derived using the free boundary approach of [10]. To do this, we investigate the problem (1.4) and, similarly to Subsection 2.2.1, make the ansatz that the optimal exercise rule is a one-sided threshold rule constituted by the first exit time from the continuation region $\left(0, y^{*}\right)$. According to the Bellman principle, we expect the candidate $G$ to be $r$-harmonic in $\left(0, y^{*}\right)$. On the other hand, on the exercise region $\left[y^{*}, \infty\right)$ the decision maker cannot exercise unless the signal process $N$ jumps. In an infinitesimal time interval $d t$, the signal process $N$ has probability $\lambda d t$ of making a jump. This means that in time $d t$, the jump and, consequently, exercise with payoff $g(x)$ has probability $\lambda d t$. On the other hand, the absence of jump forces the decision maker to wait with probability $1-\lambda d t$. Formally, this suggests with a heuristic use of Dynkin's formula, see, e.g., [11], p. 133, that

$$
\begin{aligned}
G(x) & =g(x) \lambda d t+(1-\lambda d t) \mathbf{E}_{x}\left[e^{-r d t} G\left(X_{d t}\right)\right] \\
& =\lambda g(x) d t+(1-\lambda d t)[G(x)+(\mathcal{A}-r) G(x) d t] \\
& =G(x)+(\mathcal{A}-r) G(x) d t+\lambda(g(x)-G(x)) d t
\end{aligned}
$$

for all $x>y^{*}$ under the intuition $d t^{2}=0$. Finally, this yields the condition

$$
(\mathcal{A}-(r+\lambda)) G(x)=-\lambda g(x)
$$

for all $x>y^{*}$. Moreover, we can expect that $g(x)<G(x)$ on $\left(0, y^{*}\right)$ and due to the possibility that $N$ doesn't jump when $X \geq y^{*}$ that $G(x)<g(x)$ on $\left(y^{*}, \infty\right)$. To complete the free boundary problem, we must pose a boundary condition at $y^{*}$. Following [10], we require the smooth pasting principle to hold, i.e., the candidate $G$ to be continuously differentiable over the boundary $y^{*}$. Under this condition it is elementary to check that $G\left(y^{*}\right)=g\left(y^{*}\right)$. Now we are in position to pose the free boundary problem: Determine the unique solution $\left(G, y^{*}\right)$ for the problem

$$
\begin{cases}G(0+) \geq 0, & \\ G\left(y^{*}\right)=g\left(y^{*}\right), & x<y^{*} \\ (\mathcal{A}-r) G(x)=0, \text { and } G(x)>g(x), & x>y^{*} \\ (\mathcal{A}-(r+\lambda)) G(x)=-\lambda g(x) \text { and } G(x)<g(x), & \end{cases}
$$

Assume now that a unique solution $\left(G, y^{*}\right)$ exists and that $x<y^{*}$. The condition $(\mathcal{A}-r) G(x)=0$ implies that $G$ can be expressed as $G(x)=c_{1} \psi_{r}(x)+c_{2} \varphi_{r}(x)$, where $c_{i} \geq 0$. Since we are looking for a solution that is 
bounded in the origin, we find that $c_{2}=0$. Now, let $x \geq y^{*}$. A particular solution to the fourth condition of the free boundary problem (2.16) is the resolvent $\lambda\left(R_{r+\lambda} g\right)$ and, consequently, the general solution can be written as $G(x)=\lambda\left(R_{r+\lambda} g\right)(x)+d_{1} \psi_{r+\lambda}(x)+d_{2} \varphi_{r+\lambda}(x)$. We observe that the assumptions of Theorem 1.1 imply that $d_{1}=0$. Now, the second condition in (2.16) implies that $g\left(y^{*}\right)=c_{1} \psi_{r}\left(y^{*}\right)=\lambda\left(R_{r+\lambda} g\right)\left(y^{*}\right)+d_{2} \varphi_{r+\lambda}\left(y^{*}\right)$. This in turn implies that

$$
c_{1}=\frac{g\left(y^{*}\right)}{\psi_{r}\left(y^{*}\right)}, \quad d_{2}=\frac{g\left(y^{*}\right)-\lambda\left(R_{r+\lambda} g\right)\left(y^{*}\right)}{\varphi_{r+\lambda}\left(y^{*}\right)},
$$

and, consequently, that the candidate $G$ can be expressed as

$$
G(x)= \begin{cases}\lambda\left(R_{r+\lambda} g\right)(x)+\frac{g\left(y^{*}\right)-\lambda\left(R_{r+\lambda} g\right)\left(y^{*}\right)}{\varphi_{r+\lambda}\left(y^{*}\right)} \varphi_{r+\lambda}(x), & x \geq y^{*}, \\ \frac{g\left(y^{*}\right)}{\psi_{r}\left(y^{*}\right)} \psi_{r}(x), & x<y^{*} .\end{cases}
$$

To identify the candidate for the optimal stopping threshold, we use the smooth pasting principle. Indeed, since the candidate $G$ is assumed to be continuously differentiable over the boundary $y^{*}$, we observe that the condition

$$
g\left(y^{*}\right) \frac{\psi_{r}^{\prime}\left(y^{*}\right)}{\psi_{r}\left(y^{*}\right)}-\lambda\left(R_{r+\lambda} g\right)^{\prime}\left(y^{*}\right)-\frac{g\left(y^{*}\right)-\lambda\left(R_{r+\lambda} g\right)\left(y^{*}\right)}{\varphi_{r+\lambda}\left(y^{*}\right)} \varphi_{r+\lambda}^{\prime}\left(y^{*}\right)=0
$$

must be satisfied. This can be rewritten as

$$
g\left(y^{*}\right)\left(\frac{\psi_{r}^{\prime}\left(y^{*}\right)}{\psi_{r}\left(y^{*}\right)}-\frac{\varphi_{r+\lambda}^{\prime}\left(y^{*}\right)}{\varphi_{r+\lambda}\left(y^{*}\right)}\right)=\lambda\left(R_{r+\lambda} g\right)^{\prime}\left(y^{*}\right)-\frac{\varphi_{r+\lambda}^{\prime}\left(y^{*}\right)}{\varphi_{r+\lambda}\left(y^{*}\right)} \lambda\left(R_{r+\lambda} g\right)\left(y^{*}\right) .
$$

By invoking the representation (1.2) and straightforward differentiation, we find that the right hand side can be expressed as

$$
\lambda\left(R_{r+\lambda} g\right)^{\prime}\left(y^{*}\right)-\frac{\varphi_{r+\lambda}^{\prime}\left(y^{*}\right)}{\varphi_{r+\lambda}\left(y^{*}\right)} \lambda\left(R_{r+\lambda} g\right)\left(y^{*}\right)=\lambda \frac{S^{\prime}\left(y^{*}\right)}{\varphi_{r+\lambda}\left(y^{*}\right)} \int_{y^{*}}^{\infty} \varphi_{r+\lambda}(y) g(y) m^{\prime}(y) d y .
$$

Consequently, the optimality condition (2.18) can be rewritten as

$$
\lambda \psi_{r}\left(y^{*}\right) \int_{y^{*}}^{\infty} \varphi_{r+\lambda}(y) g(y) m^{\prime}(y) d y=g\left(y^{*}\right)\left(\frac{\psi_{r}^{\prime}\left(y^{*}\right)}{S^{\prime}\left(y^{*}\right)} \varphi_{r+\lambda}\left(y^{*}\right)-\frac{\varphi_{r+\lambda}^{\prime}\left(y^{*}\right)}{S^{\prime}\left(y^{*}\right)} \psi_{r}\left(y^{*}\right)\right) .
$$

Denote as $w(x)=\frac{\psi_{r}^{\prime}(x)}{S^{\prime}(x)} \varphi_{r+\lambda}(x)-\frac{\varphi_{r+\lambda}^{\prime}(x)}{S^{\prime}(x)} \psi_{r}(x)$. It is a straightforward application of the harmonicity properties of $\psi_{r}$ and $\varphi_{r+\lambda}$ to establish that $w^{\prime}(x)=-\lambda \varphi_{r+\lambda}(x) \psi_{r}(x) m^{\prime}(x)$ for all $x \in \mathbf{R}_{+}$. Now, Fundamental Theorem of Calculus implies that

$$
w\left(y^{*}\right)=\lambda \int_{y^{*}}^{\infty} \varphi_{r+\lambda}(y) \psi_{r}(y) m^{\prime}(y) d y
$$


and, consequently, that the optimality condition (2.18) can be expressed as

$$
\psi_{r}\left(y^{*}\right) \int_{y^{*}}^{\infty} \varphi_{r+\lambda}(y) g(y) m^{\prime}(y) d y=g\left(y^{*}\right) \int_{y^{*}}^{\infty} \varphi_{r+\lambda}(y) \psi_{r}(y) m^{\prime}(y) d y
$$

Under the assumptions of Theorem 1.1, we know from Lemma 2.2 that this equation has a unique solution denoted as $x^{*}$. By combining the expressions (2.17) and (2.19), we have the same candidate for the value of the problem (1.4) and, consequently, of the problem (1.6) as we did in Subsection 2.2.1. However, we had to make a priori assumptions on the differentiability of the candidate $G$ over the optimal stopping boundary in setting up and solving the free boundary problem (2.16). This is in contrast to Subsection 2.2.1, where we formulated the variational inequalities (2.10) in terms of the resolvent operator and used its properties to identify the boundary $x^{*}$ and compute the candidates $G_{0}$ and $G$ directly without such assumptions. It is also interesting to note how different approaches suit better for different problems. Indeed, we saw how the derivation of the candidate $G_{0}$ is natural using the resolvent semigroup whereas the free boundary approach is tailor made for the derivation of the candidate $G$.

2.3. The verification phase. In the previous subsections we derived the candidates $\left(G, x^{*}\right)$ and $\left(G_{0}, x^{*}\right)$ for the solutions of the problems (1.4) and (1.6), respectively. From the point of view of the verification, the continuous time formulations (1.4) and (1.6) are not that handy. In order to remedy this, define the filtration $\mathbb{G}=\left\{\mathcal{G}_{n}\right\}_{n \geq 0}$ as $\mathcal{G}_{n}:=\mathcal{F}_{T_{n}}$ for all $n \geq 0$, where $T_{i}$ is the $i$ th jump time of the signal process $N$, and the $\mathbb{G}$-adapted process $Z$ as $Z_{n}:=\left(T_{n}, X_{T_{n}}\right)$. Moreover, define the sets $\mathcal{N}$ and $\mathcal{N}_{0}$ as

$$
\begin{aligned}
\mathcal{N} & =\{N \geq 1: N \text { is a } \mathbb{G} \text {-stopping time }\} \\
\mathcal{N}_{0} & =\{N \geq 0: N \text { is a } \mathbb{G} \text {-stopping time }\}
\end{aligned}
$$

Then Lemma 1 of [10] implies that the optimal stopping problems (1.4) and (1.6) can be formulated alternatively as

$$
\begin{aligned}
V(x) & =\sup _{N \in \mathcal{N}} \mathbf{E}\left[\tilde{g}\left(Z_{N}\right) \mid Z_{0}=(0, x)\right], \\
V_{0}(x) & =\sup _{N \in \mathcal{N}_{0}} \mathbf{E}\left[\tilde{g}\left(Z_{N}\right) \mid Z_{0}=(0, x)\right],
\end{aligned}
$$

for all $x \in \mathbf{R}_{+}$where $\tilde{g}\left(Z_{n}\right):=e^{-r T_{n}} g\left(X_{T_{n}}\right)$. Formulations (2.20) allow a straightforward usage of martingale techniques in the verification phase, as we will shortly see. We recall that the candidates $G$ and $G_{0}$ are connected via the condition $G(x)=\lambda\left(R_{r+\lambda} G_{0}\right)(x)$ for all $x \in \mathbf{R}_{+}$. Using this, we are in position to prove the following. 
Lemma 2.3. Let the assumptions of Theorem 1.1 hold. Then the process

$$
S:=\left(e^{-r T_{n}} G_{0}\left(X_{T_{n}}\right) ; \mathcal{G}_{n}\right)_{n \geq 0}
$$

is a non-negative uniformly integrable supermartingale for all initial states $X_{0}=x \in \mathbf{R}_{+}$.

Proof. Let $U$ be an exponentially distributed random time with mean $\frac{1}{\lambda}$ and independent of $X$. Then $G_{0}(x) \geq G(x)=\lambda\left(R_{r+\lambda} G_{0}\right)(x)=\mathbf{E}_{x}\left[e^{-r U} G_{0}\left(X_{U}\right)\right]$ for all $x \in \mathbf{R}_{+}$. Thus the process $S$ is a non-negative supermartingale. In order to prove uniform integrability, it suffices to show that $\sup _{n} \mathbf{E}_{x}\left[S_{n}\right]<\infty$ and $\sup _{n} \mathbf{E}_{x}\left[S_{n} \mathbf{1}_{A}\right] \rightarrow 0$ as $\mathbf{P}(A) \rightarrow 0$; then uniform integrability follows from [25], p. 190, Lemma 2.

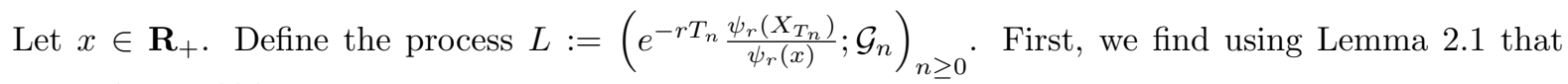
$\mathbf{E}_{x}\left[L_{1}\right]=\frac{\lambda\left(R_{r+\lambda} \psi_{r}\right)(x)}{\psi_{r}(x)}=1$. Thus, the strong Markov property of the underlying $X$ implies that $L$ satisfies $\mathbf{E}_{x}\left[L_{n}\right]=1$ for all $n \geq 0$. Now, define the measure $\mathbf{P}_{x}^{*}$ on $(\Omega, \mathcal{F})$ as

$$
\mathbf{P}_{x}^{*}(A)=\mathbf{E}_{x}\left[L_{n} \mathbf{1}_{A}\right]
$$

see [5], p. 34. Let $A \in \mathcal{F}$ and $n \geq 0$. By substituting $G_{0}$ into $S$, we find that

$$
\begin{aligned}
\frac{\mathbf{E}_{x}\left[S_{n} \mathbf{1}_{A}\right]}{\psi_{r}(x)} & =\mathbf{E}_{x}\left[\frac{G_{0}\left(X_{T_{n}}\right)}{\psi_{r}\left(X_{T_{n}}\right)} \mathbf{1}_{A} L_{n}\right] \\
& =\mathbf{E}_{x}\left[\frac{g\left(x^{*}\right)}{\psi_{r}\left(x^{*}\right)} \mathbf{1}_{A} \mathbf{1}_{\left\{X_{T_{n}}<x^{*}\right\}} L_{n}\right]+\mathbf{E}_{x}\left[\frac{g\left(X_{T_{n}}\right)}{\psi_{r}\left(X_{T_{n}}\right)} \mathbf{1}_{A} \mathbf{1}_{\left\{X_{T_{n}} \geq x^{*}\right\}} L_{n}\right]
\end{aligned}
$$

Since $\hat{x}$ is the global maximum of the function $x \mapsto \frac{g(x)}{\psi_{r}(x)}$, expression (2.21) yields

$$
0 \leq \frac{\mathbf{E}_{x}\left[S_{n} \mathbf{1}_{A}\right]}{\psi_{r}(x)} \leq \frac{g(\hat{x})}{\psi_{r}(\hat{x})}\left\{\mathbf{E}_{x}\left[\mathbf{1}_{A} \mathbf{1}_{\left\{X_{T_{n}}<x^{*}\right\}} L_{n}\right]+\mathbf{E}_{x}\left[\mathbf{1}_{A} \mathbf{1}_{\left\{X_{T_{n}} \geq x^{*}\right\}} L_{n}\right]\right\}=\frac{g(\hat{x})}{\psi_{r}(\hat{x})} \mathbf{P}_{x}^{*}(A)
$$

First, let $A=\Omega$ in the inequality (2.22). Since $\mathbf{E}_{x}\left[S_{n}\right] \leq \frac{g(\hat{x})}{\psi_{r}(\hat{x})} \psi_{r}(x)$, we find that $\sup _{n} \mathbf{E}_{x}\left[S_{n}\right]<\infty$. On the other hand, it is evident from the definition of $\mathbf{P}_{x}^{*}$ that $\mathbf{P}_{x}^{*}(A) \rightarrow 0$ whenever $\mathbf{P}_{x}(A) \rightarrow 0$. Thus, we conclude using the inequality (2.22) that $\mathbf{E}_{x}\left[S_{n} \mathbf{1}_{A}\right] \rightarrow 0$ and, consequently, that $\sup _{n} \mathbf{E}_{x}\left[S_{n} \mathbf{1}_{A}\right] \rightarrow 0$ as $\mathbf{P}_{x}(A) \rightarrow 0$.

In Lemma 2.3 we showed that under the assumptions of Theorem 1.1 the process $n \mapsto e^{-r T_{n}} G_{0}\left(X_{T_{n}}\right)$ is not only a non-negative $\mathbb{G}$-supermartingale but also uniformly integrable. Uniform integrability will be needed in the proof of the next lemma, where we use optional stopping with a stopping time that is not almost surely bounded. 
Lemma 2.4. Let the assumptions of Theorem 1.1 hold. Let $\tau_{0}^{*}=T_{N_{x^{*}}^{0}}$ where $N_{x^{*}}^{0}=\inf \left\{n \geq 0: X_{T_{n}} \geq x^{*}\right\}$. Then

$$
G_{0}(x)=\mathbf{E}_{x}\left[e^{-r \tau_{0}^{*}} g\left(X_{\tau_{0}^{*}}\right)\right]=V_{0}(x)
$$

for all $x \in \mathbf{R}_{+}$.

Proof. Coupled with Lemma 2.3, the optional sampling theorem implies that $G_{0}(x) \geq \mathbf{E}_{x}\left[e^{-r T_{N}} G_{0}\left(T_{N}\right)\right] \geq$ $\mathbf{E}_{x}\left[e^{-r T_{N}} g\left(T_{N}\right)\right]$ for all $\mathbb{G}$-stopping times $N$. Hence, $G_{0}(x) \geq V_{0}(x)$ for all $x \in \mathbf{R}_{+}$. To prove that this inequality holds as an equality, i.e., that the function $G_{0}$ can be attained by the admissible stopping rule "stop at time $\tau_{0}^{*}$, it suffices to show that the stopped process

$$
Q=\left(e^{-r T_{N_{x^{*}}^{0} \wedge n}} G_{0}\left(X_{T_{N_{x^{*}}^{0} \wedge n}}\right) ; \mathcal{G}_{n}\right)_{n \geq 0}
$$

is a martingale. We recall the definition of the process $S$ from Lemma 2.3. Now for each $n \geq 1$, we find that

$$
\mathbf{E}_{x}\left[Q_{n} \mid \mathcal{G}_{n-1}\right]=\mathbf{E}_{x}\left[S_{n} \mathbf{1}_{\left\{N_{x^{*}}^{0} \geq n\right\}} \mid \mathcal{G}_{n-1}\right]+\sum_{i=0}^{n-1} S_{i} \mathbf{1}_{\left\{N_{x^{*}}^{0}=i\right\}}
$$

Denote as $U$ an independent exponentially distributed random time with mean $\frac{1}{\lambda}$. Using the strong Markov property and the property $G(x)=\lambda\left(R_{r+\lambda} G_{0}\right)(x)$, we find that the first term on the right hand side of (2.23) can be written as

$$
\begin{aligned}
\mathbf{E}_{x}\left[S_{n} \mathbf{1}_{\left\{N_{x^{*}}^{0} \geq n\right\}} \mid \mathcal{G}_{n-1}\right] & =e^{-r T_{n-1}} \mathbf{E}_{X_{T_{n-1}}}\left[e^{-r U} G_{0}\left(X_{U}\right)\right] \mathbf{1}_{\left\{N_{x^{*}}^{0} \geq n\right\}} \\
& =e^{-r T_{n-1}} G\left(X_{T_{n-1}}\right) \mathbf{1}_{\left\{N_{x^{*}}^{0} \geq n\right\}} .
\end{aligned}
$$

Now, since $G_{0}(x)=G(x)$ when $x \leq x^{*}$, the expressions (2.23) and (2.24) imply that

$$
\mathbf{E}_{x}\left[Q_{n} \mid \mathcal{G}_{n-1}\right]=S_{n-1} \mathbf{1}_{\left\{N_{x^{*}}^{0} \geq n\right\}}+\sum_{i=0}^{n-1} S_{i} \mathbf{1}_{\left\{N_{x^{*}}^{0}=i\right\}}=Q_{n-1} .
$$

Finally, since $Q$ is also uniformly integrable, the result follows by optional sampling, i.e.,

$$
G_{0}(x)=\mathbf{E}_{x}\left[Q_{N_{x^{*}}^{0}}\right]=\mathbf{E}_{x}\left[e^{-r \tau_{0}^{*}} G_{0}\left(X_{\tau_{0}^{*}}\right)\right]=\mathbf{E}_{x}\left[e^{-r \tau_{0}^{*}} g\left(X_{\tau_{0}^{*}}\right)\right]
$$

for all $x \in \mathbf{R}_{+}$.

We proved in Lemma 2.4 that our candidates $G_{0}$ and $T_{N_{x^{*}}^{0}}$ are the optimal characteristics of the problem (1.6). We turn now back to the problem (1.4) and use Lemmas $2.3-2.4$ to prove that the candidates $G$ and $T_{N_{x^{*}}}$ are the optimal characteristics of the problem (1.4). 
Lemma 2.5. Let the assumptions of Theorem 1.1 hold. Let $\tau^{*}=T_{N_{x^{*}}}$ where $N_{x^{*}}=\inf \left\{n \geq 0: X_{T_{n}} \geq x^{*}\right\}$. Then

$$
G(x)=\mathbf{E}_{x}\left[e^{-r \tau^{*}} g\left(X_{\tau^{*}}\right)\right]=V(x)
$$

for all $x \in \mathbf{R}_{+}$.

Proof. Since the process $S$ from Lemma 2.3 is a non-negative supermartingale, we find that

$$
\mathbf{E}_{x}\left[e^{-r T_{n}} g\left(X_{T_{N}}\right)\right] \leq \mathbf{E}_{x}\left[e^{-r T_{n}} G_{0}\left(X_{T_{N}}\right)\right] \leq \mathbf{E}_{x}\left[e^{-r T_{1}} G_{0}\left(X_{T_{1}}\right)\right]=\lambda\left(R_{r+\lambda} G_{0}\right)(x)=G(x),
$$

for all $\mathbb{G}$-stopping times $N \geq 1$ and $x \in \mathbf{R}_{+}$. By taking the supremum over all such $N$, we obtain the inequality $V(x) \leq G(x)$ for all $x \in \mathbf{R}_{+}$. To prove that this inequality hold as an equality, it suffices to show that the value $G$ is attained by the admissible stopping rule "stop at time $\tau^{*}$ ". By conditioning on the first jump time $T_{1}$, we find by using the strong Markov property, Lemma 2.4, and the condition $G(x)=\lambda\left(R_{r+\lambda} G_{0}\right)(x)$ that

$$
\mathbf{E}_{x}\left[e^{-r \tau^{*}} g\left(X_{\tau^{*}}\right)\right]=\mathbf{E}_{x} \int_{0}^{\infty} e^{-r t} \mathbf{E}_{X_{t}}\left[e^{-r \tau_{0}^{*}} g\left(X_{\tau_{0}^{*}}\right)\right] \lambda e^{-\lambda t} d t=G(x),
$$

for all $x \in \mathbf{R}_{+}$.

2.4. A note on the asymptotics. We study the asymptotics of the optimal characteristics $x^{*}, V$ and $V_{0}$ as $\lambda \rightarrow 0$ and $\lambda \rightarrow \infty$. To this end, we remark that the thresholds $\hat{x}$ and $\tilde{x}$ defined in Theorem 1.1 and 1.2 are the optimal exercise thresholds for the classical continuous time stopping problems corresponding to Theorems 1.1 and 1.2 and, given that the payoff $g$ is sufficiently smooth, satisfy (uniquely) the smooth pasting conditions $g(\hat{x}) \psi_{r}^{\prime}(\hat{x})=\psi_{r}(\hat{x}) g^{\prime}(\hat{x})$ and $g(\tilde{x}) \varphi_{r}^{\prime}(\tilde{x})=\varphi_{r}(\hat{x}) g^{\prime}(\tilde{x})$, cf. [2]. Moreover, the value functions $\hat{V}$ and $\tilde{V}$ corresponding to $\hat{x}$ and $\tilde{x}$ read as

$$
\hat{V}(x)=\left\{\begin{array}{ll}
g(x), & x \geq \hat{x}, \\
\frac{g(\hat{x})}{\psi_{r}(\hat{x})} \psi_{r}(x), & x<\hat{x},
\end{array} \quad \tilde{V}(x)= \begin{cases}\frac{g(\tilde{x})}{\varphi_{r}(\tilde{x})} \varphi_{r}(x), & x>\tilde{x}, \\
g(x), & x \leq \tilde{x} .\end{cases}\right.
$$

cf. [2]. Using this notation, we prove the following result.

Proposition 2.6. Let $x^{*}, V$ and $V_{0}$ be given by Theorem 1.1. Then

(1) $x^{*}$ is an increasing function of $\lambda$,

(2) $x^{*} \rightarrow \hat{x}, V(x) \rightarrow \hat{V}(x)$ and $V_{0}(x) \rightarrow \hat{V}(x)$ as $\lambda \rightarrow \infty$,

(3) $V(x)=0$ and $V_{0}(x)=g(x)$ when $\lambda=0$,

for all $x \in \mathbf{R}_{+}$. 
Proof. First, we notice that on the limit $\lambda=0$ the signal process jumps only at $T_{0}=0$ and $T_{\infty}=\infty$ implying that $V(x)=0$ and $V_{0}(x)=g(x)$ for all $x \in \mathbf{R}_{+}$. Now, let $x \geq \hat{x}$. Since diffusions are Feller processes, we have that $\lambda\left(R_{r+\lambda} g\right) \rightarrow g$ as $\lambda \rightarrow \infty$ in sup-norm, see [21], pp. 235. By coupling this with the representation

$$
V(x)=\lambda\left(R_{r+\lambda} g\right)(x)+\left(g\left(x^{*}\right)-\lambda\left(R_{r+\lambda} g\right)\left(x^{*}\right)\right) \mathbf{E}_{x}\left[e^{-(r+\lambda) \tau_{x^{*}}}\right]
$$

(see (1.7)), we deduce that $V(x) \rightarrow g(x)-$ as $\lambda \rightarrow \infty$. Monotonicity of this convergence and continuity of $V$ across the boundary $x^{*}$ imply that $x^{*}$ increases as $\lambda$ increases and, consequently, that $x^{*} \rightarrow \hat{x}$ as $\lambda \rightarrow \infty$. Finally, we conclude that $V(x) \rightarrow \hat{V}(x)$ and $V_{0}(x) \rightarrow \hat{V}(x)$ for all $x \in \mathbf{R}_{+}$as $\lambda \rightarrow \infty$.

The following proposition can be proved completely analogously under the assumptions of Theorem 1.2.

Proposition 2.7. Let $x^{\dagger}, V$ and $V_{0}$ be given by Theorem 1.2. Then

(1) $x^{\dagger}$ is a decreasing function of $\lambda$,

(2) $x^{\dagger} \rightarrow \tilde{x}, V(x) \rightarrow \tilde{V}(x)$ and $V_{0}(x) \rightarrow \tilde{V}(x)$ as $\lambda \rightarrow \infty$,

(3) $V(x)=0$ and $V_{0}(x)=g(x)$ when $\lambda=0$,

for all $x \in \mathbf{R}_{+}$.

The results of Propositions 2.6 and 2.7 are intuitively plausible. In fact, Proposition 2.6 shows unambiguously that the optimal exercise threshold of full information case dominates the optimal exercise threshold under constrained information. This is a reasonable result and reflects the phenomenon that the decision maker will settle for less return when facing uncertainty on the length of the waiting time before the next information update. Moreover, due to the partial information on the underlying $X$, profitable moments can be missed and therefore decision maker has an incentive to lower her return requirement. Proposition 2.6 shows also that increased information on the underlying $X$ (in the sense of increased $\lambda$ ) postpones the exercise in the sense that the optimal exercise threshold increases. This again makes sense, since increased $\lambda$ results into shorter expected gaps between the observations. This means that it is less likely for decision maker to miss a profitable moment and therefore she has an incentive to increase her return requirement. To close the section, we remark that an analogous interpretation holds also for Proposition 2.7.

\section{ILLustrations}

3.1. Geometric Brownian motion and perpetual American call. In this subsection we consider the problem studied in [10], namely the perpetual American call option with underlying geometric Brownian 
motion. Let $X$ be a regular linear diffusion with the infinitesimal generator

$$
\mathcal{A}=\frac{1}{2} \sigma^{2} x^{2} \frac{d^{2}}{d x^{2}}+\mu x \frac{d}{d x}
$$

where $\mu \in \mathbf{R}$ and $\sigma>0$. The scale density $S^{\prime}$ reads as $S^{\prime}(x)=x^{-\frac{2 \mu}{\sigma^{2}}}$ and the speed density $m^{\prime}$ reads as $m^{\prime}(x)=\frac{2}{(\sigma x)^{2}} x^{\frac{2 \mu}{\sigma^{2}}}$.

The optimal stopping problem is now written as

$$
V(x)=\sup _{\tau} \mathbf{E}_{x}\left[e^{-r \tau}\left(X_{\tau}-K\right)^{+} \mathbf{1}_{\{\tau<\zeta\}}\right]
$$

where $r>0$ is the constant discount factor and $K$ is an exogenously given constant. For the sake of finiteness, we assume that $\mu<r$ and $\mu-\frac{1}{2} \sigma^{2}>0$. This guarantees that the optimal exercise thresholds are finite and are attained almost surely in a finite time. It is well known that the increasing and decreasing solutions $\psi$. and $\varphi$. can be expressed as

$$
\left\{\begin{array} { l } 
{ \psi _ { r } ( x ) = x ^ { b } , } \\
{ \varphi _ { r } ( x ) = x ^ { a } , }
\end{array} \quad \left\{\begin{array}{l}
\psi_{r+\lambda}(x)=x^{\beta} \\
\varphi_{r+\lambda}(x)=x^{\alpha}
\end{array}\right.\right.
$$

where the constants

$$
\left\{\begin{array} { l } 
{ b = ( \frac { 1 } { 2 } - \frac { \mu } { \sigma ^ { 2 } } ) + \sqrt { ( \frac { 1 } { 2 } - \frac { \mu } { \sigma ^ { 2 } } ) ^ { 2 } + \frac { 2 r } { \sigma ^ { 2 } } } > 1 , } \\
{ a = ( \frac { 1 } { 2 } - \frac { \mu } { \sigma ^ { 2 } } ) - \sqrt { ( \frac { 1 } { 2 } - \frac { \mu } { \sigma ^ { 2 } } ) ^ { 2 } + \frac { 2 r } { \sigma ^ { 2 } } } < 0 , }
\end{array} \quad \left\{\begin{array}{l}
\beta=\left(\frac{1}{2}-\frac{\mu}{\sigma^{2}}\right)+\sqrt{\left(\frac{1}{2}-\frac{\mu}{\sigma^{2}}\right)^{2}+\frac{2(r+\lambda)}{\sigma^{2}}}>1, \\
\alpha=\left(\frac{1}{2}-\frac{\mu}{\sigma^{2}}\right)-\sqrt{\left(\frac{1}{2}-\frac{\mu}{\sigma^{2}}\right)^{2}+\frac{2(r+\lambda)}{\sigma^{2}}}<0 .
\end{array}\right.\right.
$$

It is a simple computation to show that the Wronskian $B_{r+\lambda}=2 \sqrt{\left(\frac{1}{2}-\frac{\mu}{\sigma^{2}}\right)^{2}+\frac{2(r+\lambda)}{\sigma^{2}}}$. Since the payoff $g(x)=(x-K)^{+}=0$ when $x \leq K$, we find after straightforward integration that the resolvent $\lambda\left(R_{r+\lambda} g\right)$ can be written as

$$
\lambda\left(R_{r+\lambda} g\right)(x)= \begin{cases}\frac{\lambda}{r+\lambda-\mu} x-\frac{\lambda}{r+\lambda} K-\frac{2 \lambda K^{1-\alpha}}{\sigma^{2} B_{r+\lambda} \alpha(1-\alpha)} x^{\alpha}, & x>K, \\ \frac{2 \lambda K^{1-\beta}}{\sigma^{2} B_{r+\lambda}(\beta-1) \beta} x^{\beta}, & x \leq K .\end{cases}
$$

We use now Theorem 1.1 to determine the optimal exercise threshold $x^{*}$ and the optimal value functions $V$ and $V_{0}$. First, elementary integration yields

$$
J(x)=\frac{2}{\sigma^{2} \kappa} x^{-\kappa},
$$


for all $x \in \mathbf{R}_{+}$, where $\kappa=\sqrt{\left(\frac{1}{2}-\frac{\mu}{\sigma^{2}}\right)^{2}+\frac{2(r+\lambda)}{\sigma^{2}}}-\sqrt{\left(\frac{1}{2}-\frac{\mu}{\sigma^{2}}\right)^{2}+\frac{2 r}{\sigma^{2}}}>0$. Similarly we find that

$$
I(x)= \begin{cases}\frac{2}{\sigma^{2}} x^{-\beta}\left(\frac{x}{\beta-1}-\frac{K}{\beta}\right), & x>K, \\ \frac{2 K^{-(\beta-1)}}{\sigma^{2} \beta(\beta-1)}, & x<K .\end{cases}
$$

Let $x>K$. It is an elementary computation to see that $\frac{I(x)}{J(x)}=\frac{\kappa x^{-b}}{\beta(\beta-1)}(\beta x-K(\beta-1))$ and, consequently, that

$$
\frac{d}{d x}\left(\frac{I(x)}{J(x)}\right) \gtreqless 0, \text { when } x \lesseqgtr x^{*}:=\frac{b(\beta-1)}{\beta(b-1)} K .
$$

We remark that it is a straightforward computation to verify that

$$
\frac{b(\beta-1)}{\beta(b-1)}=\frac{b-\frac{r}{r+\lambda} a}{b-\frac{(r-\mu) a-\lambda}{r+\lambda-\mu}}
$$

see [10], p. 147, expression (15). Finally, using the expressions (3.2) and (1.7) we obtain the representation

$$
V(x)= \begin{cases}\frac{\lambda}{r+\lambda-\mu} x-\frac{\lambda}{r+\lambda} K+\frac{\frac{r-\mu}{r+\lambda-\mu} x^{*}-\frac{r}{r+\lambda} K}{\varphi_{r+\lambda}\left(x^{*}\right)} \varphi_{r+\lambda}(x), & x \geq x^{*}, \\ \frac{x^{*}-K}{\psi_{r}\left(x^{*}\right)} \psi_{r}(x), & x<x^{*},\end{cases}
$$

for the optimal value $V$; see [10], pp. 146-147, expressions (13), (14) and (16). Thus we have derived the results on $x^{*}$ and $V$ by Dupuis and Wang from ours. A straightforward differentiation yields

$$
\frac{d x^{*}}{d \lambda}=\hat{x} \frac{1}{\beta^{2}} \frac{d \beta}{d \lambda}=\hat{x}\left(\beta^{2} \sigma^{2} \sqrt{\left(\frac{1}{2}-\frac{\mu}{\sigma^{2}}\right)^{2}+\frac{2(r+\lambda)}{\sigma^{2}}}\right)^{-1}>0,
$$

this observation is in line with Part (1) of Proposition 2.6. Moreover, since $\beta \rightarrow \infty$ as $\lambda \rightarrow \infty$, we see immediately from the representation of $x^{*}$ that $x^{*} \rightarrow \hat{x}:=\frac{b K}{b-1}$ as $\lambda \rightarrow \infty$. Finally, since $\frac{\varphi_{r+\lambda}(x)}{\varphi_{r+\lambda}\left(x^{*}\right)}<1$ whenever $x>x^{*}$, we find after elementary manipulations that

$$
\frac{\lambda}{r+\lambda-\mu} x-\frac{\lambda}{r+\lambda} K+\frac{\frac{r-\mu}{r+\lambda-\mu} x^{*}-\frac{r}{r+\lambda} K}{\varphi_{r+\lambda}\left(x^{*}\right)} \varphi_{r+\lambda}(x) \rightarrow x-K
$$

for all $x>x^{*}$ and, consequently, that both $V(x)$ and $V_{0}(x)$ tend to

$$
\hat{V}(x)= \begin{cases}x-K, & x \geq \hat{x} \\ \frac{\hat{x}-K}{\psi_{r}(\hat{x})} \psi_{r}(x), & x<\hat{x}\end{cases}
$$

as $\lambda \rightarrow \infty$. 
To end the subsection, we illustrate graphically in Figure 2 the value functions $V, V_{0}$ and $\hat{V}$ under the parameter configuration $\mu=0.01, r=0.05, \sigma^{2}=0.1, \lambda=0.1$ and $K=1.2$.

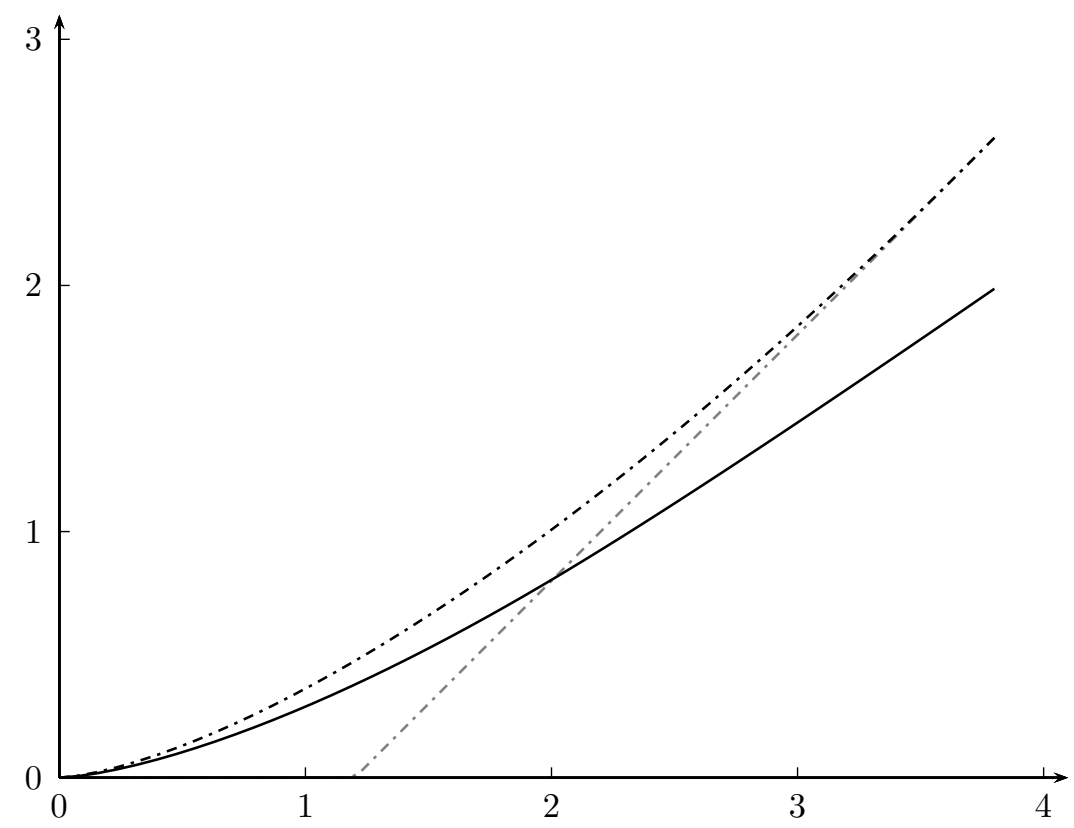

Figure 2. The value functions $\hat{V}$ under the complete information (black dashed curve) and $V$ under the information rate $\lambda=0.1$ (black solid curve) under the parameter configuration $\mu=0.01, r=0.05, \sigma^{2}=0.1$, and $K=1.2$. The grey dashed line is the payoff $g: x \mapsto(x-K)^{+}$and the value function $V_{0}$ can be recovered from the figure by first following $V$ and after the intersection the payoff $g$. The corresponding optimal thresholds are $\hat{x}=3.716$ and $x^{*}=2.010$.

3.2. Brownian motion killed in origin and perpetual American call. As an example with non-singular boundary behavior, let $X$ be a standard Brownian motion killed in the origin with the initial state $x>0$. Now, the boundary $\infty$ is natural. In this case, the functions $\psi_{r}$ and $\varphi_{r}$ read as $\psi_{r}(x)=\sinh (\sqrt{2 r} x)$ and $\varphi_{r}(x)=\exp (-\sqrt{2 r} x)$. The Wronskian $B_{r}=\sqrt{2 r}$. Moreover, the process is in natural scale, $S^{\prime}(x)=1$, that is, and the speed density reads as $m^{\prime}(x)=2$.

As in the previous subsection, the optimal stopping problem reads as

$$
V(x)=\sup _{\tau} \mathbf{E}_{x}\left[e^{-r \tau}\left(X_{\tau}-K\right)^{+} \mathbf{1}_{\{\tau<\zeta\}}\right]
$$


We verify readily that the assumptions of Theorem 1.1 are satisfied. To determine the optimal exercise threshold $x^{*}$, we compute the integrals

$$
\begin{aligned}
& g(x) \int_{x}^{\infty} \varphi_{r+\lambda}(y) \psi_{r}(y) m^{\prime}(y) d y=\frac{x-K}{\lambda} e^{-\sqrt{2(r+\lambda)} x}(\sqrt{2(r+\lambda)} \sinh (\sqrt{2 r} x)+\sqrt{2 r} \cosh (\sqrt{2 r} x)) \\
& \psi_{r}(x) \int_{x}^{\infty} \varphi_{r+\lambda}(y) g(y) m^{\prime}(y) d y=\frac{2 \sinh (\sqrt{2 r} x)}{\sqrt{2(r+\lambda)}} e^{-\sqrt{2(r+\lambda)} x}\left(x-K+\frac{1}{\sqrt{2(r+\lambda)}}\right)
\end{aligned}
$$

on $x>K$. Now, the state $x^{*}$ is characterized by the identity

$$
\left(x^{*}-K\right)\left(r+\sqrt{r(r+\lambda)} \operatorname{coth}\left(\sqrt{2 r} x^{*}\right)\right)=\frac{\lambda}{\sqrt{2(r+\lambda)}}
$$

we verify readily that the condition (3.3) has a unique root $x^{*}>K$. Furthermore, we find from (3.3) that $x^{*} \rightarrow \hat{x}$ as $\lambda \rightarrow \infty$ where $\hat{x}=\operatorname{argmax}\left\{\frac{g}{\psi_{r}}\right\}>K$ is characterized by

$$
\sqrt{2 r}(\hat{x}-K) \operatorname{coth}(\sqrt{2 r} \hat{x})=1
$$

To end the subsection, we illustrate in Figure 3 graphically the value functions $V, V_{0}$ and $\hat{V}$ under the parameter configuration $r=0.12, \lambda=1.88$, and $K=2.4$.

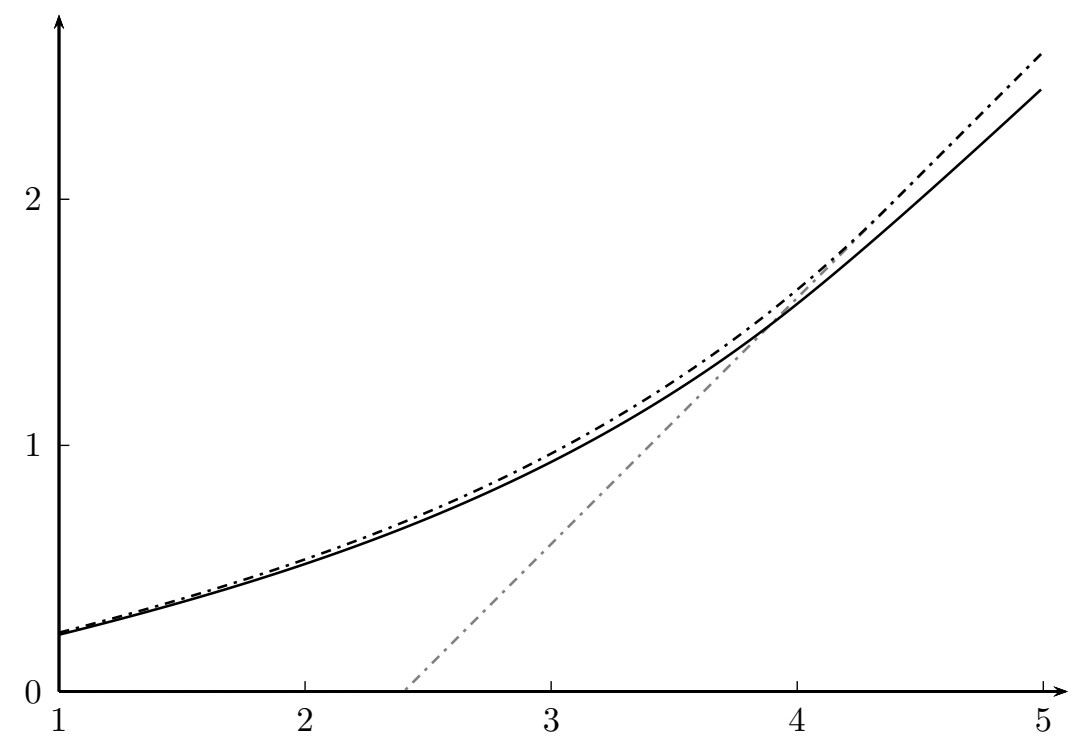

Figure 3. The value functions $\hat{V}$ under the complete information (black dashed curve) and $V$ under the information rate $\lambda=1.88$ (black solid curve). The grey dashed line is the payoff $g: x \mapsto(x-K)^{+}$and the value function $V_{0}$ can be recovered from the figure by first following the curve $V$ and then after the intersection the payoff $g$. The corresponding optimal stopping thresholds are $\hat{x}=4.386$ and $x^{*}=3.887$.

3.3. Logistic diffusion and perpetual American put. As a generalization of the geometric Brownian setting and an illustration of Theorem 1.2, we consider the case of perpetual American put with a mean 
reverting underlying $X$. More precisely, let $X$ follow a regular linear diffusion with the infinitesimal generator

$$
\mathcal{A}=\frac{1}{2} \sigma^{2} x^{2} \frac{d^{2}}{d x^{2}}+\mu x(1-\gamma x) \frac{d}{d x}
$$

where the exogenous constants $\mu, \gamma, \sigma \in \mathbf{R}_{+}$. This process is called the logistic diffusion (or the geometric Ornstein-Uhlenbeck process [17] or the radial Ornstein-Uhlenbeck process [5]) and was made famous in literature of real options at the latest by [9]. As above, a straightforward computation yields the scale density $S^{\prime}(x)=x^{-\frac{2 \mu}{\sigma^{2}}} e^{\frac{2 \gamma \mu}{\sigma^{2}} x}$ and, consequently, the speed density $m^{\prime}(x)=\frac{2}{(\sigma x)^{2}} x^{\frac{2 \mu}{\sigma^{2}}} e^{-\frac{2 \gamma \mu}{\sigma^{2}} x}$ for all $x \in \mathbf{R}_{+}$. The optimal stopping problem is now formulated as

$$
V(x)=\sup _{\tau} \mathbf{E}_{x}\left[e^{-r \tau}\left(K-X_{\tau}\right)^{+} \mathbf{1}_{\{\tau<\zeta\}}\right]
$$

with $\mu<r$ and $K>0$.

We use Theorem 1.2 to study the optimal exercise threshold $x^{\dagger}$ and the optimal value functions $V$ and $V_{0}$. We know from the literature, see, [17], Lemma 3.4.3 or [8], Section 6.5, that the decreasing solution $\varphi_{r}$ and the increasing solution $\psi_{r+\lambda}$ can be expressed as

$$
\left\{\begin{array}{l}
\varphi_{r}(x)=x^{b} U\left(b, 2 b+\frac{2 \mu}{\sigma^{2}}, \frac{2 \mu \gamma}{\sigma^{2}} x\right), \\
\psi_{r+\lambda}(x)=x^{\beta} M\left(\beta, 2 \beta+\frac{2 \mu}{\sigma^{2}}, \frac{2 \mu \gamma}{\sigma^{2}} x\right),
\end{array}\right.
$$

where $\beta=\left(\frac{1}{2}-\frac{\mu}{\sigma^{2}}\right)+\sqrt{\left(\frac{1}{2}-\frac{\mu}{\sigma^{2}}\right)^{2}+\frac{2(r+\lambda)}{\sigma^{2}}}$ and the functions $M: \mathbf{R}_{+} \rightarrow \mathbf{R}_{+}$and $U: \mathbf{R}_{+} \rightarrow \mathbf{R}_{+}$are the two linearly independent solutions of the Kummer's equation, i.e., the so-called confluent hypergeometric functions of first and second kind, cf. [1], pp. 504. Due to the analytically demanding nature of the functions $\varphi_{r}$ and $\psi_{r+\lambda}$, we will now fix a parameter setting and illustrate our results numerically and graphically. In Table 1 we present the optimal stopping thresholds for different rates $\lambda$ under the parameter configuration $\mu=0.01, r=0.05, \sigma^{2}=0.1, \gamma=0.5$, and $K=2.4$.

\begin{tabular}{|c|ccccccccc|}
\hline$\lambda$ & 0.005 & 0.1 & 0.5 & 1 & 5 & 10 & 50 & 250 & $\infty$ \\
\hline$x^{\dagger}$ & 1.837 & 1.626 & 1.248 & 1.150 & 1.023 & 0.994 & 0.956 & 0.939 & 0.926 \\
\hline
\end{tabular}

Table 1. The optimal stopping threshold $x^{\dagger}$ for various information rates $\lambda$ and the smooth pasting threshold $\tilde{x}(\lambda=\infty)$ of ordinary the ordinary case under the parameter configuration $\mu=0.01, r=0.05, \sigma^{2}=0.1$, $\gamma=0.5$, and $K=2.4$.

The numerical results reported in Table 1 are in line with our main results. In particular, these numerics indicate that the optimal exercise threshold $x^{\dagger}$ is a decreasing function of the intensity $\lambda$ and that these thresholds tend to the smooth pasting threshold $\tilde{x}$ of the ordinary case as $\lambda$ increases. To end the subsection, 
we illustrate graphically in Figure 4 the value functions $V, V_{0}$ and $\tilde{V}$ under the parameter configuration $\mu=0.01, r=0.05, \sigma^{2}=0.1, \gamma=0.5, \lambda=0.1$ and $K=2.4$.

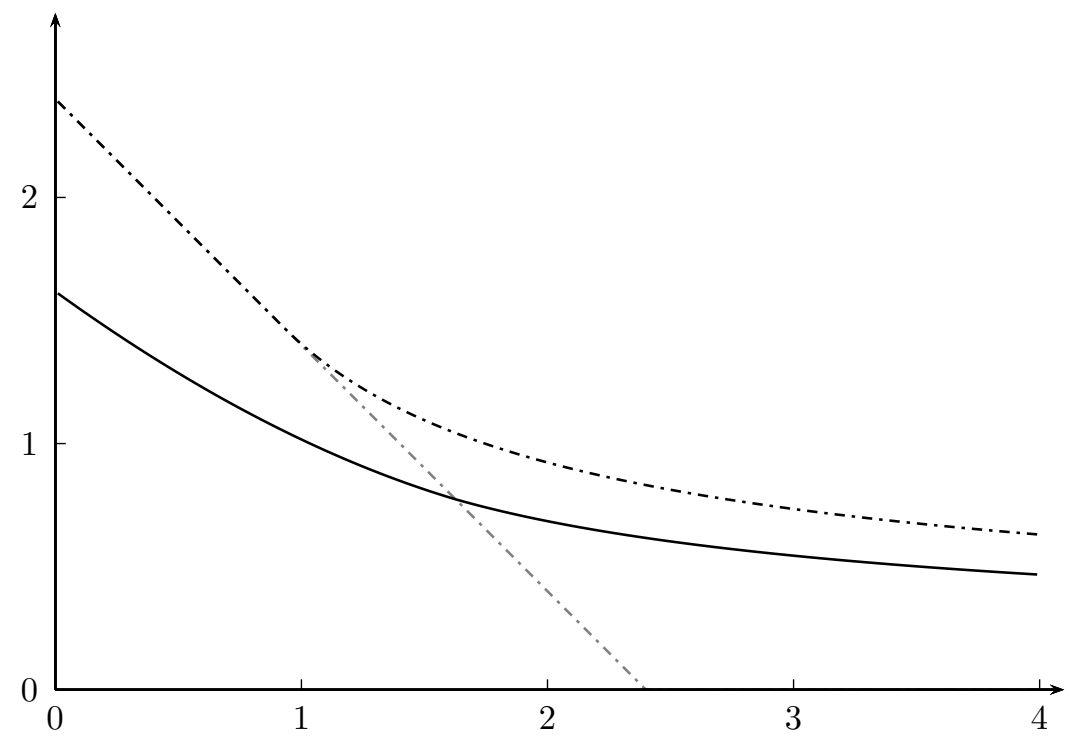

Figure 4. The value functions $\tilde{V}$ under the complete information (black dashed curve) and $V$ under the information rate $\lambda=0.1$ (black solid curve). The grey dashed line is the payoff $g: x \mapsto(K-x)^{+}$and the value function $V_{0}$ can be recovered from the figure by first following the payoff $g$ and then after the intersection the curve $V$. The corresponding optimal stopping thresholds are $\tilde{x}=0.926$ and $x^{\dagger}=1.626$.

3.4. CEV process and perpetual American put. As another illustration of Theorem 1.2, we consider the perpetual American put when the underlying dynamics follow a CEV process $X$ with the differential generator $\mathcal{A}=\frac{1}{2} x^{4} \frac{d^{2}}{d x^{2}}$ and the initial state $x>0$. This process is a classical example of an Itô integral which is a strict local martingale and it is connected to a theory of financial bubbles, see, e.g., [7]. The boundaries of the state space are classified as follows: the origin is natural and $\infty$ is entrance, see, e.g., [15]. Now, the functions $\psi_{r}$ and $\varphi_{r}$ read as $\psi_{r}(x)=x \exp \left(-\sqrt{2 r} x^{-1}\right)$ and $\varphi_{r}(x)=x \sinh \left(\sqrt{2 r} x^{-1}\right)$. Moreover, the process $X$ is in natural scale and the density of the speed measure reads as $m^{\prime}(x)=\frac{2}{x^{4}}$. Finally, the Wronskian $B_{r}=\sqrt{2 r}$.

As in the previous subsection, the optimal stopping problem is written as

$$
V(x)=\sup _{\tau} \mathbf{E}_{x}\left[e^{-r \tau}\left(K-X_{\tau}\right)^{+} \mathbf{1}_{\{\tau<\zeta\}}\right],
$$


with $r, K>0$. We use Theorem 1.2 to find the optimal characteristics of this problem. To determine the optimal stopping threshold $x^{\dagger}$, we compute the integrals

$$
\begin{aligned}
& g(x) \int_{0}^{x} \psi_{r+\lambda}(y) \varphi_{r}(y) m^{\prime}(y) d y=\frac{K-x}{\lambda} e^{-\sqrt{2(r+\lambda)} x^{-1}}\left(\sqrt{2(r+\lambda)} \sinh \left(\sqrt{2 r} x^{-1}\right)+\sqrt{2 r} \cosh \left(\sqrt{2 r} x^{-1}\right)\right) \\
& \varphi_{r}(x) \int_{0}^{x} \psi_{r+\lambda}(y) g(y) m^{\prime}(y) d y=\frac{2 \sinh \left(\sqrt{2 r} x^{-1}\right)}{\sqrt{2(r+\lambda)}} e^{-\sqrt{2(r+\lambda)} x^{-1}}\left(K-x+\frac{K x}{\sqrt{2(r+\lambda)}}\right)
\end{aligned}
$$

on $x<K$. After elementary manipulations, we find that the threshold $x^{\dagger}$ is characterized by the condition

$$
\left(K-x^{\dagger}\right)\left(\frac{2 r}{\sqrt{2(r+\lambda)}} \sinh \left(\sqrt{2 r} x^{\dagger^{-1}}\right)+\sqrt{2 r} \cosh \left(\sqrt{2 r} x^{\dagger-1}\right)\right)=\frac{\lambda}{r+\lambda} K x^{\dagger} \sinh \left(\sqrt{2 r} x^{\dagger-1}\right)
$$

again, we verify readily that the equation (3.7) has a unique root $x^{\dagger}<K$. Furthermore, we observe that $x^{\dagger} \rightarrow \tilde{x}$ as $\lambda \rightarrow \infty$ where $\tilde{x}=\operatorname{argmax}\left\{\frac{g}{\varphi_{r}}\right\}<K$ is characterized by

$$
\sqrt{2 r}(K-\tilde{x}) \operatorname{coth}\left(\sqrt{2 r} \hat{x}^{-1}\right)=K \tilde{x}
$$

To end the subsection, we illustrate in Figure 5 graphically the value functions $V, V_{0}$ and $\tilde{V}$ under the parameter configuration $r=0.05, \lambda=1$, and $K=2.4$.

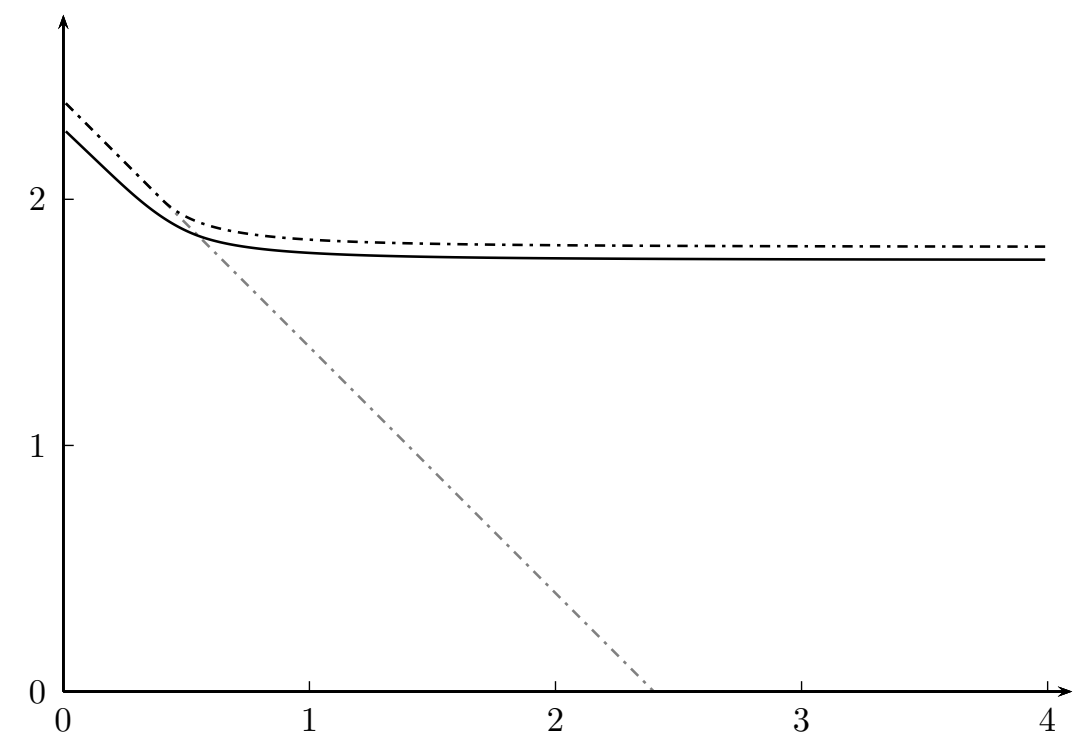

Figure 5. The value functions $\tilde{V}$ under the complete information (black dashed curve) and $V$ under the information rate $\lambda=1$ (black solid curve). The grey dashed line is the payoff $g: x \mapsto(K-x)^{+}$and the value function $V_{0}$ can be recovered from the figure by first following the payoff $g$ and then after the intersection the curve $V$. The corresponding optimal stopping thresholds are $\tilde{x}=0.400$ and $x^{\dagger}=0.548$. 


\section{Concluding Comments}

We studied in this paper the optimal stopping problems (1.4) and (1.6) proposed originally by Dupuis and Wang in [10]. In [10], the authors solve these problems in the case of perpetual American call with underlying geometric Brownian motion. As our main result, we proposed a mild set of conditions on the underlying and the payoff and solved the problems under these conditions. These results are formulated in Theorems 1.1 and 1.2. After proving the necessary auxiliary results, we proposed in Subsection 2.2.1 the variational equalities (2.10) and (2.13) and solved them directly using the Markovian theory of linear diffusions. As a result, we produced candidates (2.14) and (2.12) for the optimal solutions. We also derived these candidates using the free boundary approach of [10] and established that the approaches are consistent. The verification phase was carried out in Section 3 in the spirit of [10]. In [10], the authors interpret the signal process $N$ as an exogenous liquidity constraint. In this paper, we proposed and discussed an alternate interpretation of $N$ as an exogenous information constraint.

The main contribution of this paper is that it generalizes considerably the results of [10] with respect to the underlying and the payoff structure. In comparison to [2], Theorem 3, we made additional assumptions on the limiting behavior of these functions and on the integrability of the payoff $g$. However, these additional assumptions are not very restrictive from the applications point of view. In this sense, this study shows that the introduction of an independent Poissonian signal process $N$ to the problem lowers the degree of solvability of the problem only slightly. Moreover, we avoided making any a priori differentiability assumptions in deriving the candidates in Subection 2.2.1. In fact, we saw that the smoothness properties of the values can be seen as a consequence of the variational inequalities.

Acknowledgements: An anonymous referee is gratefully acknowledged for careful reading and for a number of very helpful comments. The author thanks Prof. Luis H.R. Alvarez, Prof. Fred Espen Benth, Dr. Paul C. Kettler, Prof. Andreas E. Kyprianou and Prof. Paavo Salminen for discussions and comments on earlier versions of this paper. Financial support from Research Foundation of OP-Pohjola group and the project "Electricity markets: modelling, optimization and simulation (EMMOS)" funded by the Norwegian Research Council under grant 205328/v30 is acknowledged. Prof. Esko Valkeila and Department of Mathematics and System Analysis in Aalto University School of Science and Technology are acknowledged for hospitality.

\section{REFERENCES}

[1] Abramowitz M. and Stegun I. Handbook of Mathematical Functions, 1968, Dover Publications 
[2] Alvarez, L. H. R. Reward Functionals, Salvage Values and Optimal Stopping, 2001, Mathematical Methods of Operations Research, 54/2, $315-337$

[3] Alvarez, L. H. R. and Stenbacka R. Adoption of Uncertain Multi-Stage Technology Projects: A Real Options Approach, 2001, Journal of Mathematical Economics, 35, 71 - 97

[4] Alvarez, L. H. R. and Stenbacka R. Takeover Timing, Implementation Uncertainty, and Embedded Disinvestment Options, 2006, Review of Finance, 10, 417 - 441

[5] Borodin, A. and Salminen P. Handbook on Brownian Motion - Facts and Formula, 2002, Birkhäuser, Basel

[6] Boyarchenko, S. and Levendorskii, S. Exit Problems in Regime-Switching Models, 2008, Journal of Mathematical Econom$i c s, 44,180-206$

[7] Cox, A. M. G. and Hobson, D. G. Local Martingales, Bubbles and Option Prices, 2005, Finance and Stochastics, 9, 477 492

[8] Dayanik, S. and Karatzas, I. On the Optimal Stopping Problem for One-Dimensional Diffusions, 2003, Stochastic Processes and Their Applications, 107/2, $173-212$

[9] Dixit, A. and Pindyck, R. Investment Under Uncertainty, 1994, Princeton University Press

[10] Dupuis, P. and Wang, H. Optimal Stopping with Random Intervention Times, 2002, Advances in Applied Probability, 34, $141-157$

[11] Dynkin, E. Markov processes I, 1965, Springer-Verlag

[12] Guo, X. An Explicit Solution to an Optimal Stopping Problem with Regime Switching, 2001, Journal of Applied Probability, $38,464-481$

[13] Guo, X. and Liu, J. Stopping at the Maximum of Geometric Brownian Motion when Signals are Received, 2005, Journal of Applied Probability, 42, $826-838$

[14] Guo, X., Miao, J. and Morellec, E. Irreversible Investment with Regime Shift, 2005, Journal of Economic Theory, 122, 37 $-59$

[15] Jeanblanc, M., Yor, M., and Chesney, M. Mathematical Methods for Financial Markets, 2009, Springer Verlag London

[16] Jiang, Z. and Pistorius M. R. On Perpetual American Put Valuation and First-Passage In a Regime-Switching Model With Jumps, 2008, Finance and Stochastics, 12, 331 - 355

[17] Johnson, T. C. The Optimal Timing of Investment Decisions, 2006, Ph.D Thesis, King's College, London

[18] Lempa, J. A Note on Optimal Stopping of Diffusions with a Two-sided Optimal Rule, 2010, Operations Research Letters, $38,11-16$

[19] Øksendal, B. Stochastic Differential Equations, 5th Edition, 2000, Springer

[20] Peskir, G. and Shiryaev, A. Optimal Stopping and Free Boundary Problems, 2006, Birkhäuser, Basel

[21] Rogers, L. C. G. and Williams, D. Diffusions, Markov Processes and Martingales: Volume 1, 2001, Cambridge university press

[22] Rogers, L. C. G. and Zane, O. A Simple Model of Liquidity Effects, 2002, Advances in Finance and Stochastics: Essays in Honour of Dieter Sondermann, Springer, 161 - 176

[23] Salminen, P. Optimal Stopping of One-Dimensional Diffusions, 1985, Matematische Nachrichten, 124,85 - 101

[24] Shepp, L and Shiryaev, A. The Russian Option: Reduced Regret, 1993, Annals of Applied Probability, 3 , 631 - 640

[25] Shiryaev, A. Probability, 1996, 2nd Edition, Springer 